\title{
SHOCK PROPAGATION INTO \\ INHOMOGENEOUS MEDIA
}

by

James D. Strachan

B.Sc., University of British Columbia, 1968

A THESIS SUBMITTED IN PARTIAL FULFILMENT OF THE REQUIREMENTS FOR THE DEGREE OF MASTER OF SCIENCE

in the Department

of

PHYSICS

We accept this thesis as conforming to the required standard

THE UNIVERSITY OF BRITISH COLUMBIA

September, 1969 
In presenting this thesis in partial fulfilment of the requirements for an advanced degree at the University of British Columbia, 1 agree that the Library shall make it freely available for reference and study. I further agree tha permission for extensive copying of this thesis for scholarly purposes may be granted by the Head of my Department or by his representatives. It is understood that copying or publication of this thesis for financial gain shall not be allowed without my written permission.

Department of $\rho_{H Y S / C S}$

The University of British Columbia Vancouver 8 , Canada

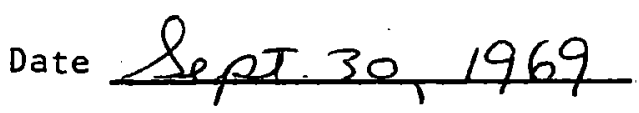




\section{ABSTRACT}

The propagation of a shock wave into a general inhomogeneous flow field is studied. The equations for the shock velocity through such a region are developed in a general manner. A consequence of these equations is the development of the shock wave as a probe into unknown flow fields. The shock velocity is measured and the initial parameters ahead of the shock are calculated. The unique advantage of the shock probe is that it does not perturb the gas ahead of the front.

An experimental application is described in which the shock probe is used to analyze the unknown flow field created by a constricted arc light source. The flow field is subsequently identified as a radiation front at the Chapman-Jouguet point. 
TABLE OF CONTENTS

Page

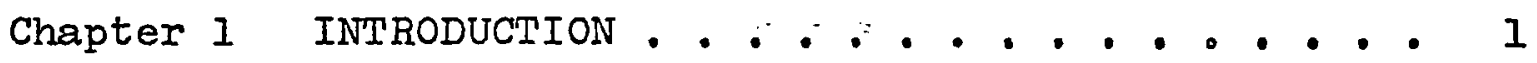
BASIC SHOCK THEORY . . . . . . . 3

Chapter 2 SHOCK PROPAGATION INTO A DISCONTINUOUS

INHOMOGENEOUS MEDIUM ......... 8

Chapter 3 SHOCK PROPAGATION INTO A CONTINUOUS

INHOMOGENEOUS MEDIUM ........ 14

Chapter 4 PROBING A FLOW FIELD WITH A SHOCK WAVE . . . 25

Chapter 5 CONCLUSIONS . . . . . . . . 44 BIBLIOGRAPHY ............ 47

Appendix A THE DOUBLY REFLECTED WAVE . . . . . . . 49 Appendix B PARAMETERS AT THE BOUNDARY . . . . . . 52 Appendix C CONTINUOUSLY VARYING PARAMETERS . . . . . 67 Appendix D THE SHOCK TUBE . . . . . . . . . 74 Appendix E OTHER INTERESTING ASPECTS . . . . . . 86 Appendix F THE EXPERIMENTAL SET-UP . . . . . . . 90 Appendix G PIEZOELECTRIC PROBE CALIBRATION . . . . 93 


\section{LIST OF ILLUSTRATIONS}

Figure

Page

1 Shock Wave in Shock Frame ......... 3

2 Effective Adiabatic Constant for Argon . . . 6

3 Shock - Shock Collision .......... 10

4 Shock Polar ............. II

5 Thermal Discontinuity - Shock Collision . . . 12

6 Shock Polars ............ 13

7 Shock Crossing an Infinitessimal Discontinuity 17

8 Shock as Viewed by Observer Changing Frames . . 24

9 Shock - Flow Field Intersection . . . . . 27

10 Shock - Flow Field Intersection Enlarged . . 29

11 Shock - Flow Field Intersection Enlarged . . 30

12 Shock Wave Probing the Flow Field . . . . 34

$13 p_{3}$ vs Distance............. 36

$14 \mu_{3}$ vs Distance............. 36

$15 W_{3}$ vs Distance ............ 37

$16 \rho_{3}$ vs Distance............... 38

17 Radiation Front Velocity vs Downstream Pressure 39

$18 \mu_{3}$ Behind the Radiation Front ....... 40

19. $\rho_{3}$ Behind the Radiation Front ....... 40

20 Pressure Behind the Radiation Front ...... 41

21 Pressure Behind the Radiation Front ...... 41

22 Radiation Front at Low Downstream Pressure . . 45 
Figure

A Doubly Reflected Wave . . . . . . . . 50

B-1 Graphical Solution for Intersection Problem . . 54 B-2 Error Bounds on State 4. . . . . . . . 55

c Shock Velocity Behind Radiation Front . . . 70

D-1 Contact Surface Velocity . . . . . . . . 77

D-2 Contact Surface Velocity . . . . . . . . 77

D-3 Vertical Smear Camera . . . . . . . 79

D-4 Shock Velocity vs $V_{c}$. . . . . . . 80

D-5 Shock Attenuation . . ... . . . . . 81

D-6 Shock Attenuation ............. . 82

D-7 Shock Attenuation . . . . . . . . 82

D-8 Crowbar Results . . . . . . . . . . . 84

D-9 Crowbar Results . . . . . . . . . 85

E-1 Radiation Effects in the Shock Heated Gas . : 87

E-2 Radiation Energy Addition at a Shock Front - 88

F-l Apparatus . . . . . . . . . . . . 91

F-2 Smear Camera - . - - . - . - . . . 92

G Piezoelectric Probe Calibration . . . . . 93 


\section{ACKNOWLEDGEMENTS}

I am sincerely indebted to Dr. B. AhIborn for his encouragement and his stimulating insight which was the motivation for this thesis.

I am grateful for the many interesting discussions with all members of the Plasma Physics Group, and in particular I thank J.P. Huni and R. Ardila. R. Ardila performed the pressure measurements which gave us confidence in the shock probe.

I am also grateful to the National Research Council for their financial assistance. 
Chapter 1

INTRODUCTION

The propagation of shock waves into uniform gases is well understood, but no general study has attempted to understand the propagation of shocks into inhomogeneous and moving media.

As a background, one needs to know the essentials of standard shock wave theory which are briefly outlined in the first chapter. Quite generally, the properties of the inhomogeneous media can change either abruptly or slowly. Shock propagation into abrupt changes has been thoroughly studied and is outlined in the second chapter. The propagation of a shock into slowly varying initial conditions is presented in the third chapter and is one of the main contributions of this thesis.

From the theoretical study of the penetration of shocks into inhomogeneous media, we draw one general conclusion which is discussed in chapter four. We conclude that it is possible to probe an unknown flow field with a shock wave of known strength. By measuring the local variation of the shock front velocity one can obtain the local variations of the initial parameters. Three shocks of different strengths are needed to encounter the reproducible but unknown flow field in order that the initial pressure, density, and particle velocity can be derived. An experimental appli- 
cation which probes an unknown flow field is described in chapter four.

All standard calculations and detailed descriptions are relegated to the appendix, of which, appendix $D$ may be of special interest to someone working with the same type of shock tube, and appendix $E$ which discusses other interesting aspects will be fascinating for everyone. 
When the velocity of a fluid becomes comparable with or exceeds that of sound, effects due to the compressibility of the fluid become important. One of the most distinctive features of supersonic flow is that shock waves (strong compression waves) can occur in it. Thermodynamically, a shock wave is a discontinuous jump in pressure, density, temperature, and entropy which, in the frame of the shock front, separates uniform equilibrium conditions of subsonic flow from uniform equilibrium conditions of supersonic flow. Consider one dimensional plane flow in a frame at which the shock front is at rest and label the quantities before the shock front as 1 and those behind the shock front as 2 (fig. 1 ).

\section{SHOCK DIRECTION}

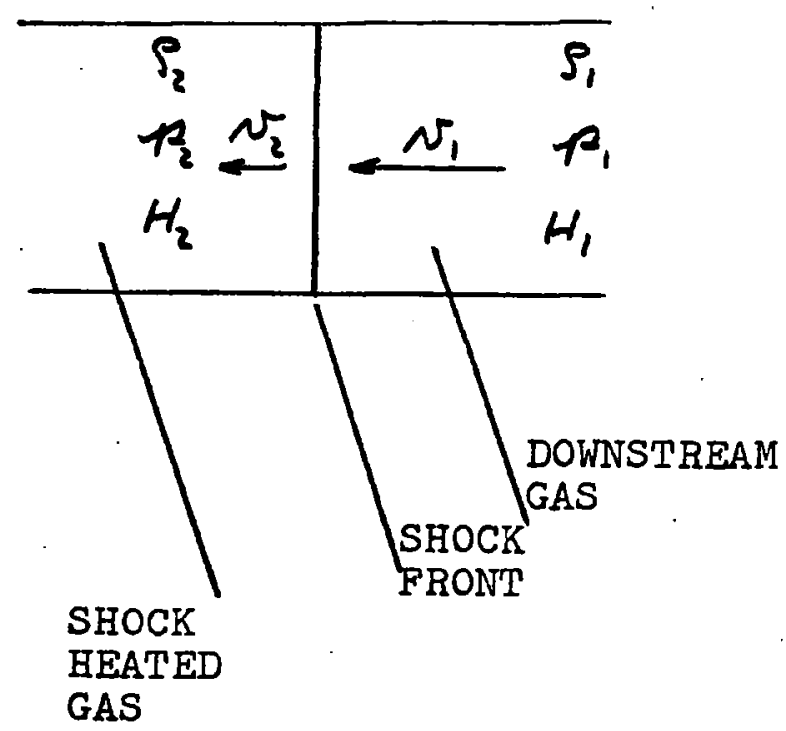

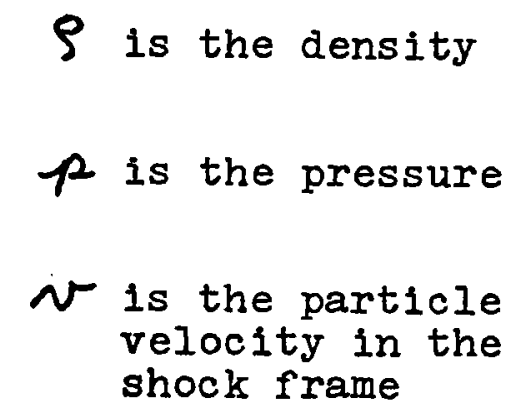

$H$ is the enthalpy of a unit mass of gas

Fig. I Shock Wave in the Shock Frame 
The three conservation equations can be written as follows :

1. Conservation of Mass

$$
\rho_{1} v_{1}=\rho_{2} v_{2}
$$

2. Conservation of Momentum

$$
p_{1}+\rho_{1} v_{1}^{2}=p_{2}+\rho_{2} v_{2}^{2}
$$

3. Conservation of Energy

$$
H_{1}+\frac{1}{2} v_{1}^{2}=H_{2}+\frac{1}{a} v_{2}^{2}
$$

These conservation equations together with the equation of state are sufficient to determine the solution in terms of the shock parameters $\frac{P_{2}}{p_{1}}, \frac{\rho_{2}}{\rho_{1}}, \frac{T_{2}}{T_{1}}$ and since the initial conditions ahead of the shock front are known; $P_{2}, \rho_{2}$, and $T_{2}$ are determined once the shock front velocity is measured.

For an ideal gas the equation of state is

$$
H=\frac{\gamma}{\gamma-1} \frac{p}{\rho}
$$

where $\gamma$ is the ratio of specific heats and is a constant ( $5 / 3$ for argon). The corresponding solutions are 
$-5-$

$$
\begin{aligned}
\frac{p_{2}}{p_{1}} & =\frac{\partial \gamma M^{2}-(\gamma-1)}{\gamma+1} \\
\frac{\rho_{2}}{\rho_{1}} & =\frac{(\gamma+1) \dot{M}^{2}}{(\gamma-1) M^{2}+2} . \\
\frac{T_{2}}{T_{1}} & =\frac{\left(\gamma M^{2}-\frac{\gamma-1}{2}\right)\left(\frac{\gamma-1}{2} M^{2}+1\right)}{\left(\frac{\gamma+1}{2}\right)^{2} M^{2}}
\end{aligned}
$$

where $M=\frac{N_{1}}{a_{1}}$ is the Mach Number and $a$ is the speed of sound.

For monatomic gases ionization and electronic excitation are the only processes which cause a departure from ideality. In order to take ionization into account, the energy equation is usually changed so that

$$
H_{2}-H_{1}=\frac{1}{2} v_{1}^{2}-\frac{1}{2} v_{2}^{2}-\Delta E_{I}
$$

where $\Delta E_{I}$ is the change in energy due to ionization (Gaydon and Hurle, 1963). The other common way to take ionization into account is to introduce an effective adiabatic exponent $g$ which is defined by

$$
H=\frac{g}{g-1} \frac{p}{\rho}
$$

so that $g=g(p, T)$ becomes a function of pressure and 


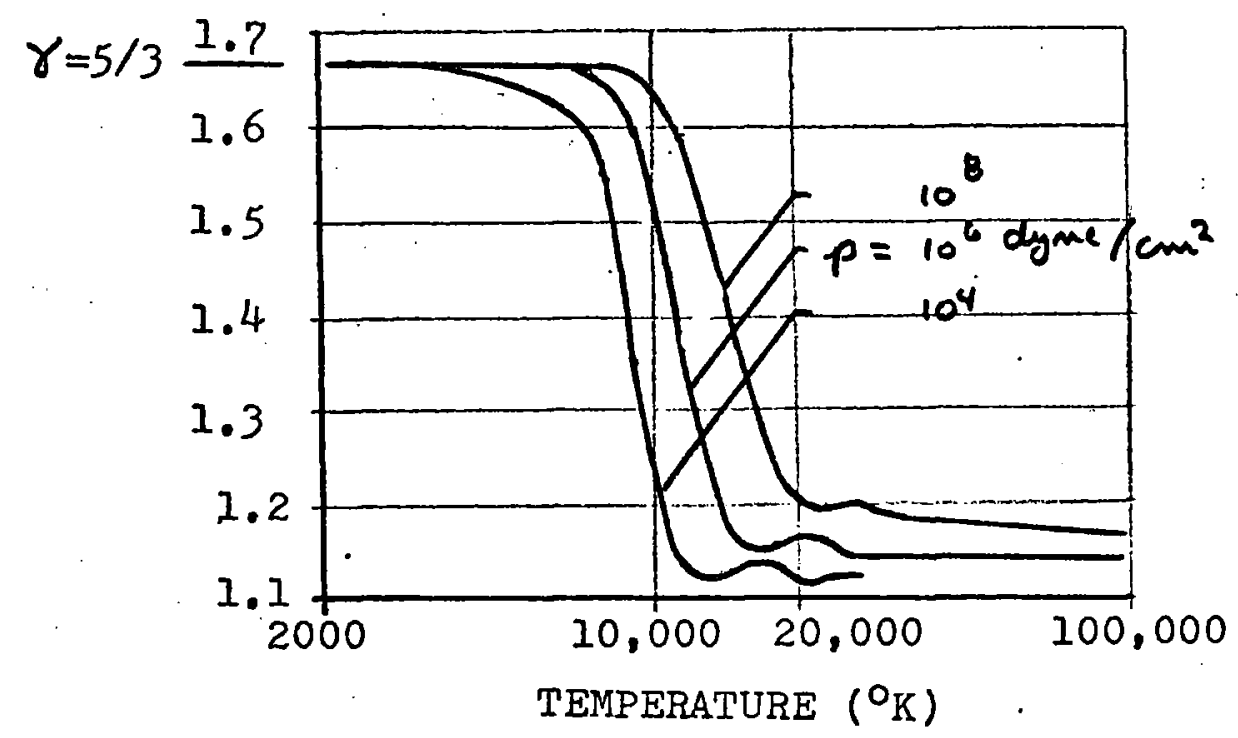

Fig. 2. Effective Adiabatic

Constant For Argon

temperature which may be calculated for any gas (fig. 2) and is, for instance, tabulated by Ahlborn and Salvat (1967).

We use the effective adiabatic exponent which has the advantage of separating the thermodynamic calculations from the hydrodynamic calculations. The hydrodynamics is contained in the Rankine - Hugoniot equations (I), (2), and (3), while the thermodynamics is confined to determining $g(p, T)$ with the inclusion of all essential lonization and excitation processes.

The exact solution of the Rankine - Hugonlot equations (Ahlborn and Salvat, 1967) now becomes 
$-7-$

$$
\begin{aligned}
& \frac{v_{2}}{v_{1}}=\frac{\rho_{1}}{\rho_{2}}=1+\frac{\frac{g_{2}}{g_{1} m_{2}-1}}{g_{2}+1}\{i+\sqrt{1+\epsilon}\} \\
& \frac{p_{2}}{p_{1}}=1-g_{1} M^{2} \frac{\frac{g_{2}}{g_{1} M^{2}-1}}{g_{2}+1}\{1+\sqrt{1+\epsilon}\}
\end{aligned}
$$

where

$$
\epsilon=\frac{2\left(g_{2}+1\right)\left(g_{1}-g_{2}\right) M^{2} g_{1}}{\left(g_{1}-1\right)\left(g_{2}-g_{1} M^{2}\right)^{2}}
$$

The temperature is found numerically from the equation of state, $T=T(g, p, \rho)$, for the particular gas under study, assuming local thermal equilibrium. 
Chapter 2

SHOCK PROPAGATION INTO A DISCONTINUOUS

INHOMOGENEOUS MEDIUM

Historically, a great deal of work has been done on the interaction of shock waves with contact surfaces in various oblique configurations (Taub, 1947). And too, the interaction of shock waves with shock waves has been well understood. This chapter is a retalloring of known theory to suit our particular interests.

Suppose a shock runs through a region in which some or all of the initial conditions vary discontinuously. There are only two possibilities --

1. either the pressure is identical on both sides of the discontinuity, or

2. the pressure jumps across the discontinuity.

If the pressure is continuous, then the discontinuity is a contact surface separating two different gases or one gas at two different temperatures. Since diffusion effects must always occur at contact surfaces they may not be truly discontinuous. Thus, contact surfaces are treated in chapter 3.under continuous inhomozeneous media and the treatment is such that the solution holds even if a contact surface were ideally discontinuous.

If the pressure changes discontinuously, the same general conservation equations must hold as held for a 
shock wave (chapter 1). Since the discontinuity surface is more general than an adiabatic shock wave, source terms must be included in the Rankine - Hugoniot equations. But to uncomplicate matters, mass source terms and force terms will be ignored. Only an energy source term is considered. In actual flow fields, mass sources at the edge of a pressure discontinuity seem very unlikely while external force terms appearing only at the edge of the discontinuity are possible but very uncommon. Energy source terms, on the other hand, occur in radiation fronts, detonation waves, and in radiation losses at shock fronts.

The conservation equations for the general pressure discontinuity become

$$
\begin{gathered}
\rho_{1} v_{1}=\rho_{2} v_{2} \\
p_{1}+\rho_{1} v_{1}^{2}=p_{2}+\rho_{2} v_{2}^{2} \\
\frac{g_{1}}{g_{1}-1} \frac{p_{1}}{\rho_{1}}+\frac{1}{2} v_{1}^{2}+\frac{w}{\rho_{1} v_{1}}=\frac{g_{2}}{g_{2}-1} \frac{\rho_{2}}{\rho_{2}}+\frac{1}{2} v_{2}^{2}
\end{gathered}
$$

where $W$ is a constant and is the energy input per unit mass. These equations have been solved exactly (Ahlborn, 1963) and the solutions are

$$
\frac{v_{2}}{v_{1}}=\frac{\rho_{1}}{g_{2}}=1+\frac{\frac{g_{2}}{g_{1} M^{2}}-1}{g_{2}+1}\left\{1+\sqrt{1+\epsilon-\frac{2\left(g_{2}^{2}-1\right) W}{g_{1} p_{1} M^{2}\left(\frac{g_{2}}{g_{M}}-1\right)^{2}}}\right\}
$$




$$
\frac{p_{2}}{p_{1}}=1-g_{1} M^{2} \frac{\frac{g_{2}}{g_{1} m^{2}-1}}{g_{2}+1}\left\{1+\sqrt{1+\epsilon-\frac{2\left(g_{2}^{2}-1\right) w}{g_{1} p_{1} m^{2}\left(\frac{g_{2}}{g_{1} m^{2}-1}\right)^{2}}}\right\}(19)
$$

Equations (18) and (19) quite generally describe the variation of pressure and density across a thermal discontinuity. We want to consider what happens when a shock front collides with a discontinulty across which equations (18) and (19) hold. As a starting point, we consider what happens when two one-dimensional plane shocks collide (Shapiro, 1954). See figure 3. We label the various equilibrium regions by numbers and denote the interface between two such regions, $i$ and $j$, as $\langle i, j\rangle$.



Fig. 3 Shock - Shock Collision 
In the above notation --

$\langle 1,3\rangle$ is the shock wave propagating towards the left into region 1 .

$\langle 1,2\rangle$ is the shock wave propagating towards the right into region 1 .

$\langle 3,5\rangle$ is the shock travelling towards the right into the already shock-heated gas in region 3 .

$\langle 2,4\rangle$ is the shock travelling towards the left into the already shock-heated gas in region 2.

$\langle 4,5\rangle$ is a contact surface which separates the two doubly shock-heated regions. The pressure and particle velocity are continuous across contact surfaces, and in this case, the contact surface velocity equals the particle velocity in regions 4 and 5 .



Fig. 4 Shock Polar 
The problem is completely specified once the initial velocities of $\langle 1,3\rangle$ and $\langle 1,2\rangle$ are known and is conveniently represented on a Shock Polar (fig. 4). The lines connecting the various states are unique and are obtained by using the Rankine - Hugoniot equations with the shock velocity as a parameter. The states 4 and 5 are determined as the intersection of the polars from states 2 and 3 .

Now we return to the more general case where the shock, $\langle 1,2\rangle$, runs into a general pressure discontinuity, $\langle 1,3\rangle$, and the situation appears as in figure 5.

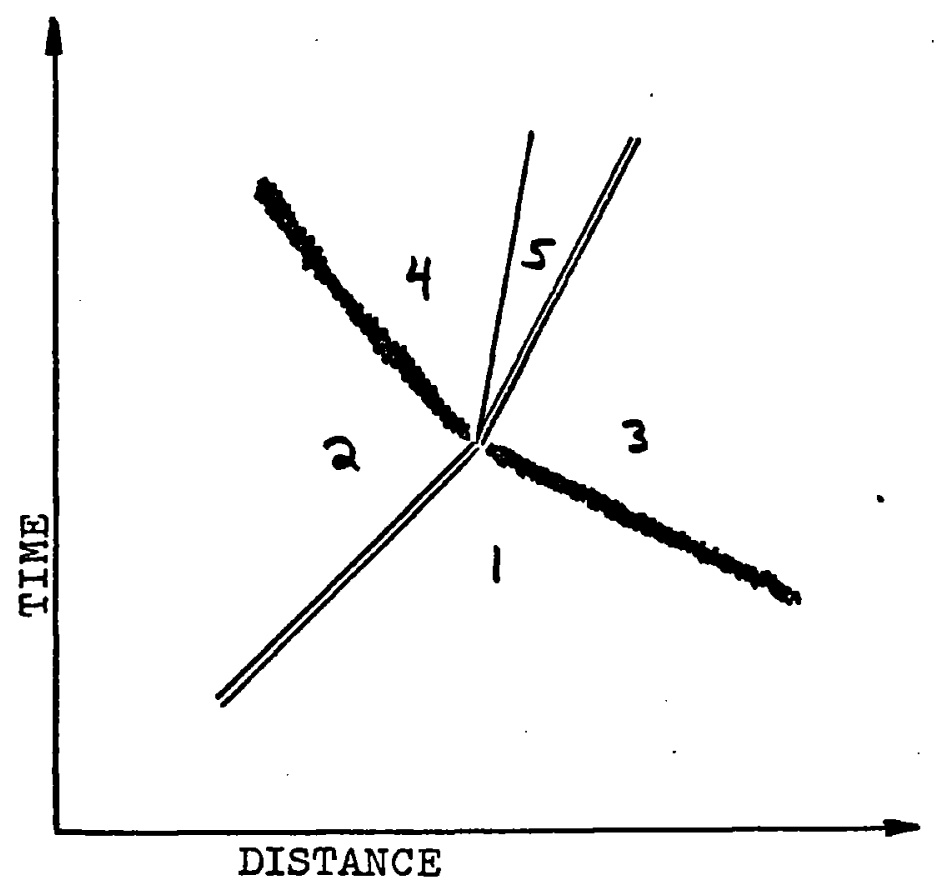

P18. 5 Thermal Discontinuity - Shock Collision 
$\langle 1,2\rangle,\langle 3,5\rangle$, and $\langle 4,5\rangle$ are as previousiy defined. But $\langle 1,3\rangle$ is a pressure discontinuity with. energy input $W_{3}$ and $\langle 2,4\rangle$ is a pressure discontinuity with energy input $W_{4}$. If these energy inputs are known, then there are fourteen unknowns: $\rho_{2}, \mu_{2}, \rho_{2}, \rho_{3}$, $\mu_{3}, \rho_{3}, \rho_{4}, \mu_{4}, \rho_{4}, V_{\langle 2,4\rangle}, \rho_{5}, \mu_{5}, p_{5}$, $V_{\langle 3,5\rangle}$; with fourteen equations: four sets of the three Rankine - Hugoniot equations plus $P_{4}=P_{5}$ and $\mu_{4}=\mu_{5}$. Therefore, it is possible to find $V_{{ }_{3}, 5}$, and probably the best method is to again draw the shock polars (fig. 6). Instead of applying the shock polar from state 1 to state 3 , use the shock polar with energy input $W_{3}$, and similarly, in going from state 2 to state 4 use the shock polar with energy input $W_{4}$.



Fig. 6 Shock Polars 


\section{Chapter 3 \\ SHOCK PROPAGATION INTO A CONTINUOUS}

INHOMOGENEOUS MEDIUM

The solution for continuously varying initial pressure and density distributions has been attacked with the view of understanding certain astrophysical phenomena such as colliding stars (De Young and Axford, 1967) and the emission of mass from the surface of stars (Nadezhin and FrankKamenetskii, 1965). The usual methods have been the chisnell method (Chisnell, 1955; Ôno, Sakashita, and Yamazaki, 1960) and the Whitham Rule (Whitham, 1958) which requires the writing of the characteristic equation. The method developed here is a generalization of the Chisnell method.

We consider the propagation of a shock wave through a general one-dimensional, continuous, and inhomogeneous medium, where the initial density, pressure, and particle velocity each vary as a function of position in a certain region. The propagation of a certain initial shock through the region is physically well defined and a unique physical process occursrthat is, the velocity remains single valued throughout the inhomogeneous region.

The shock wave velocity is, in general, a function of the initial density, inftial pressure, initial particle velocity, and the driving mechanism. The shock velocity is completely specified if these variables are precisely given. Usually, 
of course, the exact nature of the driving mechanism is not clearly known, and thus the shock velocity is usually taken as a parameter. Still, the shock velocity can be written in functional form;

$$
V=V\left(S_{1}, p_{1}, \mu_{1}, \xi\right)
$$

where $\xi$ corresponds, in some sense, to the driving mechanism. If we assume that the driving mechanism is constant, the rate of change of the shock velocity throughout the inhomogeneous region is given by differentiating equation (20),

$$
\frac{d V}{d x}=\frac{\partial V}{\partial \rho_{1}} \frac{d \rho_{1}}{d x}+\frac{\partial V}{\partial p_{1}} \frac{d p_{1}}{d x}+\frac{\partial V}{\partial \mu_{1}} \frac{d \mu_{1}}{d x}
$$

where $\rho_{1}, \rho_{1}$, and $\mu_{1}$ are treated as completely independent parameters, in line with the initial assumption of a completely general flow field. Integrating equation (21) yields

$$
V(x)=V(0)+\int_{x=0}^{x}\left(\frac{\partial V}{\partial \rho_{1}} d \rho_{1}+\frac{\partial V}{\partial p_{1}} d p_{1}+\frac{\partial V}{\partial \mu_{1}} d \mu_{1}\right)
$$

The problem of finding the shock velocity as a function of position in the inhomogeneous region has been reduced to the problem of finding three functions: 
1. $\frac{\partial V}{\partial \rho_{1}}$ at constant $p_{1}$ and $\mu_{1}$
2. $\frac{\partial V}{\partial p_{1}}$ at constant $\rho_{1}$ and $\mu_{1}$
3. $\frac{\partial v}{\partial u_{1}}$ at constant $p_{1}$ and $\rho_{1}$

Consider the physical situations which correspond to the above partial derivatives. The mathematical problem of finding

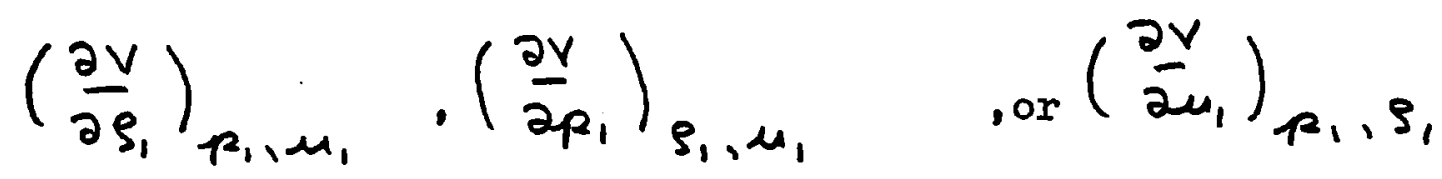

is equivalent to the physical problem of finding the motion of a normal shock wave through a non-uniform, one-dimensional medium of continuously changing density, pressure, or particle velocity. If we follow the method of Chisnell, the non-uniform region is regarded as a succession of small density, pressure, or particle velocity discontinuities separated by uniform regions.

Assume that the initial parameter. increases monotonically with distance in a certain region and is uniform outside this region. A plane shock moves in the $x$-direction through the region $x<0$ with constant strength and uniform flow behind 1t. When the shock passes through the region of the changing parameter, its strength changes and a wave is reflected backwards from 1t. In addition, the motion of the reflected wave through the non-uniform region generates another "doubly reflected " wave moving in the same direction as the incident shock. The mathematical complications encountered by consider- 
ing the doubly reflected wave are enormous, so we will limit the usefulness of our results by making the approximation that this doubly reflected wave can be ignored (appendix A).

First of all, the physical situation to consider in deriving $\frac{\partial V}{\partial S}$, is that of an infinitessimal density discontinuity (fig, 7). The contact discontinuity, $\langle 1,5\rangle$, has no discontinuity in pressure or fluid velocity, although there is an infinitessimal jump in the density, such that

$$
\rho_{s}=\rho_{1}+d \rho
$$

while

$$
\begin{array}{ll}
p_{3}=p_{4} \quad ; \quad p_{1}=p_{5} \\
\mu_{3}=\mu_{4} \quad ; \quad \mu_{1}=\mu_{5}
\end{array}
$$

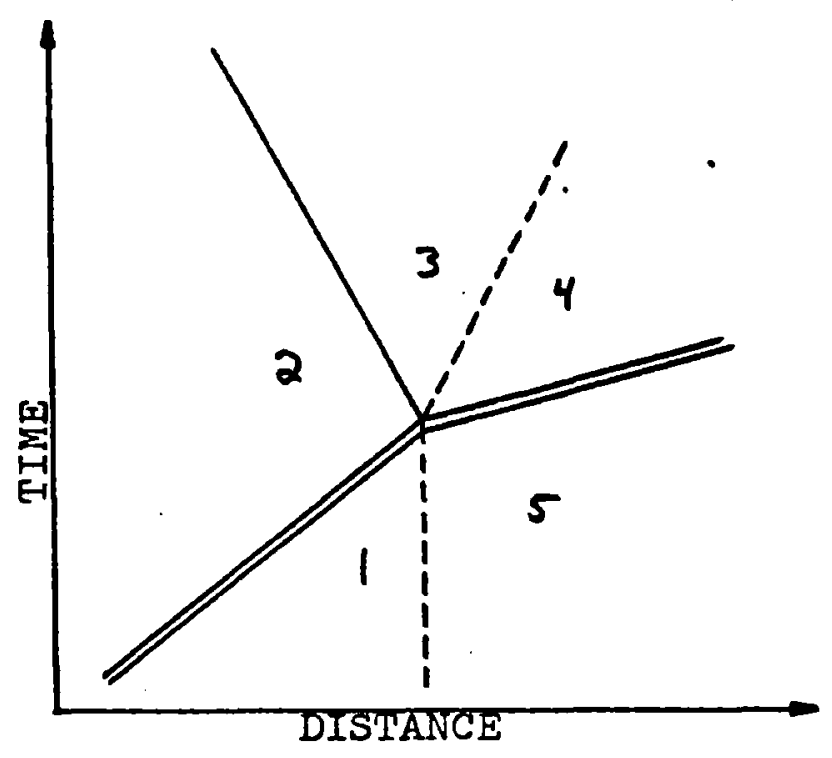

Fig. 7 Shock Crossing an Infinitessimal Discontinuity 
$-18$

The strength of the disturbance is taken as $Z_{\mathrm{mm}}$ where $z$ is the ratio of the pressure in region $m$ to that in region $m$. Therefore, the pressure ratio of the incident shock is $z_{12}=\frac{p_{2}}{p_{1}}$. By expressing the Rankine - Hugoniot equations in terms of the parameter $z$ and then solving, we obtain

$$
\begin{aligned}
& \rho_{2}=\rho_{1} \frac{\lambda^{2}+z_{12}}{1+\lambda^{2} z_{12}} \\
& \mu_{2}=\mu_{1}+\phi\left(z_{12}, p_{1}, \rho_{1}\right) \\
& V=\mu_{1}+\sqrt{\frac{p_{1}}{\rho_{1}} \frac{\lambda^{2}+z_{12}}{1-\lambda^{2}}} \\
& \lambda^{2}=\frac{g_{2}-1}{g_{2}+1}
\end{aligned}
$$

where

and

$$
\phi=\left(z_{12}-1\right) \sqrt{\frac{p_{1}}{s_{1} \frac{1-\lambda^{2}}{\lambda^{2}+z_{12}}}}
$$

Similar equations hold for the elements of the reflected wave, and differ with those for a shock only in third and higher powers of $z-1$, where $z$ is the strength of the disturbance. Provided this restriction is remembered, the shock equations may be used for the small disturbances to save formulating a second set of equations. 
Returning to the conditions on the velocity and pressure (equations 24 and 25), obtain

$$
\begin{aligned}
\phi\left(z_{12}, p_{1}, \rho_{1}\right) & -\phi\left(z_{23}, p_{2}, \rho_{2}\right) \\
& =\phi\left(z_{23}, p_{5}, \rho_{5}\right) \\
z_{12} z_{23} & =z_{54}
\end{aligned}
$$

Note that the increase in strength of the penetrated shock must be infinitessimal so that

$$
z_{54}=z_{12}+\alpha z
$$

By inserting equations (30), (31), (26), and (23) into equation (29) and taking it to the first order in smallness in terms of $\alpha \rho$ and $\alpha z$, obtain

$$
\frac{1}{S_{1}} \frac{d \rho}{d z}=\frac{2}{z_{12}-1}-\frac{1}{\lambda^{2}+z_{12}}+\frac{2}{z_{12}-1} \sqrt{\frac{1+\lambda^{2} z_{12}}{z_{12}\left(1+\lambda^{2}\right)}}
$$

but, from equation (28)

$$
V=\mu_{1}+\sqrt{\frac{\rho_{1}}{\rho_{1}} \frac{\left(\lambda^{2}+z_{12}\right)}{1-\lambda^{2}}}
$$

and differentiating with respect to $\rho_{1}$ at constant $p_{1}$ and $\mu_{1}$, obtain 
$-20-$

$$
\begin{aligned}
\left(\frac{\partial V}{\partial \rho_{1}}\right)_{p_{1}, \mu_{1}}= & \frac{1}{2} \sqrt{\frac{p_{1}}{\rho_{1}} \frac{\lambda^{2}+z}{1-\lambda^{2}}} \\
& {\left[\frac{p_{1}}{\rho_{1}\left(1-\lambda^{2}\right)} \frac{d z}{d \rho_{1}}-\frac{p_{1}}{\rho_{1}^{2}} \cdot\left(\frac{\lambda^{2}+z}{1+\lambda^{2}}\right)\right] }
\end{aligned}
$$

Therefore,

$$
\begin{aligned}
\frac{\partial V}{\partial \rho_{1}} & =-\frac{V-\mu_{1}}{2 \rho_{1}} \\
& +\frac{\rho_{1}}{2 \rho_{1}^{2}\left(1-\lambda^{2}\right)\left(V-\mu_{1}\right)\left[\frac{\partial}{z-1}-\frac{1}{z+\lambda^{2}}+\frac{\partial}{z-1} \sqrt{\frac{1+\partial^{2} z}{z\left(1+\lambda^{2}\right)}}\right]}
\end{aligned}
$$

where

$$
z=\frac{\rho_{1}}{p_{1}}\left(1-\lambda^{2}\right)\left(v-\mu_{1}\right)^{2}-\lambda^{2}
$$

so that finally

$$
\left(\frac{\partial V}{\partial \rho_{1}}\right)_{p_{1}, \mu_{1}}=f\left(\rho, p_{1}, \mu_{1}, v\right)
$$

which is Chisnell's result.

The physical situation to consider in deriving $\frac{\partial V}{\partial p_{1}}$, is that of an infinitesimal pressure discontinuity across which the particle velocity and density are continuous. Although a rather unnatural type of discontinuity, imagine some force field maintaining it until the shock wave crosses it, after which time the force field is removed. 
$-2 I-$

Again, as in figure 7 , an incident shock wave generates at the jump position $\langle 1,5\rangle$, a reflected wave $\langle 3,2\rangle$, a penetrated shock $\langle 5,4\rangle$, and a contact surface $\langle 3,4\rangle$. The physical quantities before and behind the shock are connected by the Rankine - Hugoniot equations ( 18 to 28). The following boundary equations also apply:

$$
\begin{aligned}
\mu_{1} & =\mu_{5} \\
\mu_{3} & =\mu_{4} \\
\rho_{1} & =\rho_{5} \\
p_{3} & =p_{4} \\
p_{5} & =p_{1}+d p
\end{aligned}
$$

And, as previously

$$
\begin{gathered}
\phi\left(z_{12}, p_{1}, s_{1}\right)-\phi\left(z_{23}, p_{2}, s_{2}\right) \\
=\phi\left(z_{54}, p_{5}, s_{5}\right)
\end{gathered}
$$

and

$$
z_{12} z_{23}=z_{15} z_{45}
$$

where

$$
z_{45}=z_{12}+d z
$$


$-22-$

Therefore,

$$
z_{12} z_{23}=\left(1+\frac{d p_{1}}{p_{1}}\right)\left(z_{12}+d z\right)
$$

or

$$
z_{23}=\left(1+\frac{\alpha p_{1}}{p_{1}}\right)\left(1+\frac{\alpha z}{z_{12}}\right)
$$

and by taking to the first order of smallness,

$$
z_{23}=1+\frac{d p_{1}}{p_{1}}+\frac{d z}{z_{12}}
$$

By inserting equations (37 to 41 , and 47) into equation (42),

$$
\frac{d z}{d p_{1}}=\frac{\sqrt{z \frac{1+\lambda^{2} z}{1+\lambda^{2}}}-\frac{z-1}{2}}{p_{1}\left(1-\sqrt{\frac{1+\lambda^{2} z}{z\left(1+\lambda^{2}\right)}}-\frac{z-1}{\partial\left(\lambda^{2}+z\right)}\right)}
$$

Differentiating equation (28),

$$
\frac{\partial V}{\partial p_{1}}=\left(v-\mu_{1}\right)\left\{\frac{1}{\partial p_{1}}+\frac{1}{\partial\left(\lambda^{2}+z\right)} \frac{d z}{d p_{1}}\right\}
$$

and inverting (28),

$$
z={\frac{\rho}{\rho_{1}}}_{\rho_{1}}\left(1-\lambda^{2}\right)\left(v-\mu_{1}\right)^{2}-\lambda^{2}
$$


then by entering equations (48) and (50) into equation (49), obtain

$$
\left(\frac{\partial V}{\partial p_{1}}\right)_{\rho_{1}, \mu_{1}}=h\left(p_{1}, \rho_{1}, \mu_{1}, V\right)
$$

Equation (51) has been derived in a manner very smilar to the previous derivation of $\frac{\partial V}{\partial \rho}$ by the Chisnell method.

Finaliy, the physical situation corresponding to the partial derivative $\frac{\partial V}{\partial \mu}$, is a discontinuity surface across which only the initial particle velocity changes. An analagous experimental situation would be for an observer to watch the propagation of a shock wave from some inertial frame at which $\mu_{1}=\mu_{1}^{\prime}$. The observer sees the shock propagation with some velocity $V^{\prime}$ and with strength parameters $z^{\prime}$ and $\bar{\rho}_{1}$. Then, at time $T=0$ let the observer change to a different frame-- one in which the particle velocity is

$$
\mu_{1}=\mu_{1}^{\prime}+\alpha \mu_{1}
$$

where $d \mu$, is non-relativistic.

To that observer just described, the flow of the shock wave appears as in figure 8. Since the only process to occur In figure 8 has been that of the observer changing frames, the shock will have the same strength parameters $z$ and $\frac{\rho_{2}}{\rho_{1}}$. even though the shock velocity has changed. The change in shock velocity is $\alpha \mu_{1}$, and therefore

$$
\left(\frac{\partial V}{\partial u_{1}}\right)_{p_{1}, \rho_{1}}=1
$$




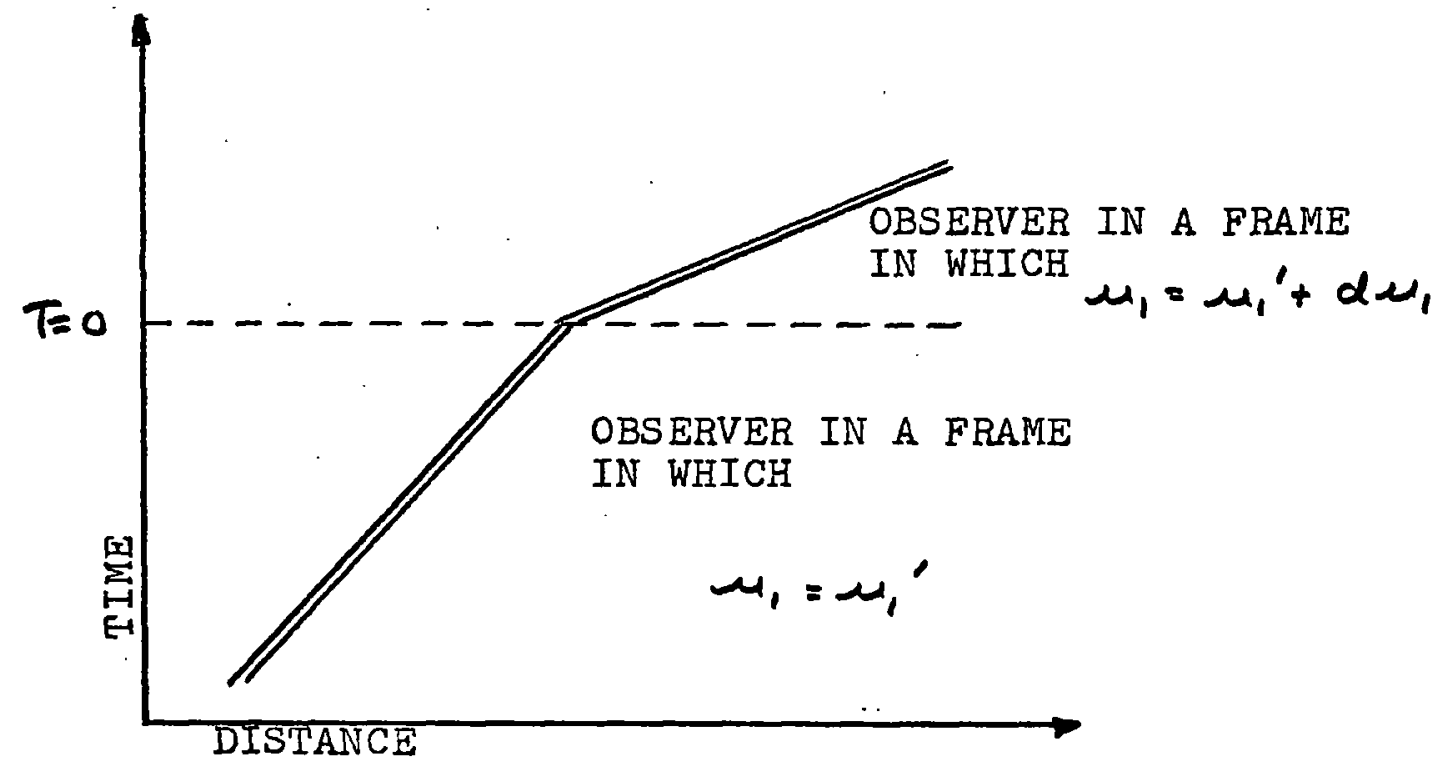

Fig. 8 SHOCK AS VIEWED BY OBSERVER CHANGING FRAMES

All the partial derivatives have finally been calculated and the integral expression for the velocity as a function of position is

$$
\begin{aligned}
V(x)=V(0) & +\mu_{1}(x)-\mu_{1}(0) \\
& +\int_{x=0}^{x} f\left(\rho_{1}, p_{1}, \mu_{1}, V\right) d \rho_{1} \\
& +\int_{x=0}^{x} h\left(\rho_{1}, p_{1}, \mu_{1}, V\right) d p_{1}
\end{aligned}
$$

This equation gives the attenuation of the original shock front velocity $V(0)$ after traveling a distance $X$ into a general inhomogeneous region. 


\section{Chapter 4 \\ PROBING A FLOW FIELD WITH A}

SHOCK WAVE.

The study of shock propagation into inhomogeneous media has an interesting application. Since one knows the relation between the initial conditions and the final velocity, a measurement of the shock front velocity can be used to diagnose an unknown plasma flow field. Most methods of measuring the parameters in an unknown flow field depend upon, either measurements of the radiation given off, or probes which are assumed to perturb the flow field by a negligible amount. We chose the other extreme and let a shock wave interact with the unknown flow field. The shock is a large amplitude perturbation, but its interaction with any variations in a flow field is understood from chapters two and three. The unique advantage of this probing technique lies in the fact that the unknown flow field does not experience the probing tool (the shock wave) until the measurement is completed. This phenomenon is a result of the shock wave travelling faster than the signal velocity (speed of sound) in the unknown medium. Thus, a technique which relies upon measurement of the shock front velocity will always be measuring the properties of the unperturbed gas, since the shock always flows into unperturbed gas.

Suppose the parameters in a supersonic flow field are unknown. A supersonic flow fleld will be bounded by a discontinuity of the type discussed in chapter two. The parameters 
immediately behind the boundary can be obtained by inverting the discussion in chapter two and measuring the velocities of the four fronts, $\langle 1,2\rangle,\langle 1,3\rangle,\langle 2,4\rangle$, and $\langle 3,5\rangle$. By observing the variation of the shock velocity throughout the unknown flow field and inverting the results of chapter three, the dependence of the plasma parameters on position can be obtained throughout the flow field.

In our experiment, we use the shock wave to investigate the properties of the flow fleld produced by a Bogen light source. Ahlborn and Zuzak $(1968,1969)$ utilized the Bogen light source to create a radiation front behind a window. In our case we removed the window to observe the flow fleld created by the light source. One important question is raised, "is the flow field a radiation front created by the intense ultraviolet radiation from the light source, or is it a blast wave, perhaps radiation supported, driven by the escaping arc heated gas from the light source?" Details of the experimental set-up are presented in appendix F. The shock waves are formed by a hybrid electro-thermal-magnetic shock tube designed by P.R. Smy (appendix D), and the measuring device is a smear camera. The shock wave and flow field from the light source collide head-on and the smear camera is at right angles to the direction of flow (fig. 9).

The experiment requires intersections of the flow fleld created by the light source, with shock waves. This is quite a simple procedure. Different shock strengths are acquired by 



Fig. 9 Shock-- Flow Field Intersection 
changing the spark gap separation, $S_{1}$, in the driver section and thus changing the capacitor bank firing voltage. The intersection position of the flow field and the shock wave are. altered by adusting the relative delay setting on the delay unlts for the shock driver and Bogen light souroe, which are triggered by the rotating mirror of the smear camera.

With such an easy experiment, there was nothing to do but to proceed. First, by using colour film, some initial results were obtained (fig. 9 and 11). In figure 9, the shock wave (in blue from the left) intersects an invisible preceding shock from the light source (in green from the right). On many of the pictures, the separation of the shock wave from the main Iuminous mass is visible (due to impurity radiation) and the separation occurs where the flow fleld changes colour from white to blue in figure 9. $\left(T_{2}\right)$

In figure 11 the intersection is seen in greater detail since the slit on the smear camera has been greatly reduced. The photograph is very interesting because all the regions that were discussed qualitatively in figure 3 (chapter 2) can be identified. The regions $1,2,3,4,5$ in figure 10 are precisely those indicated in figure 3.

$\langle 1,2\rangle$ and $\langle 1,3\rangle$ are shock waves. $\langle 2,4\rangle$ and $\langle 3,5\rangle$ are the refracted shock waves. $\langle 4,5\rangle$ is the contact surface separating the two doubly heated shock regions. This surface is visible because of the temperature difference across $\langle 4,5\rangle$. 

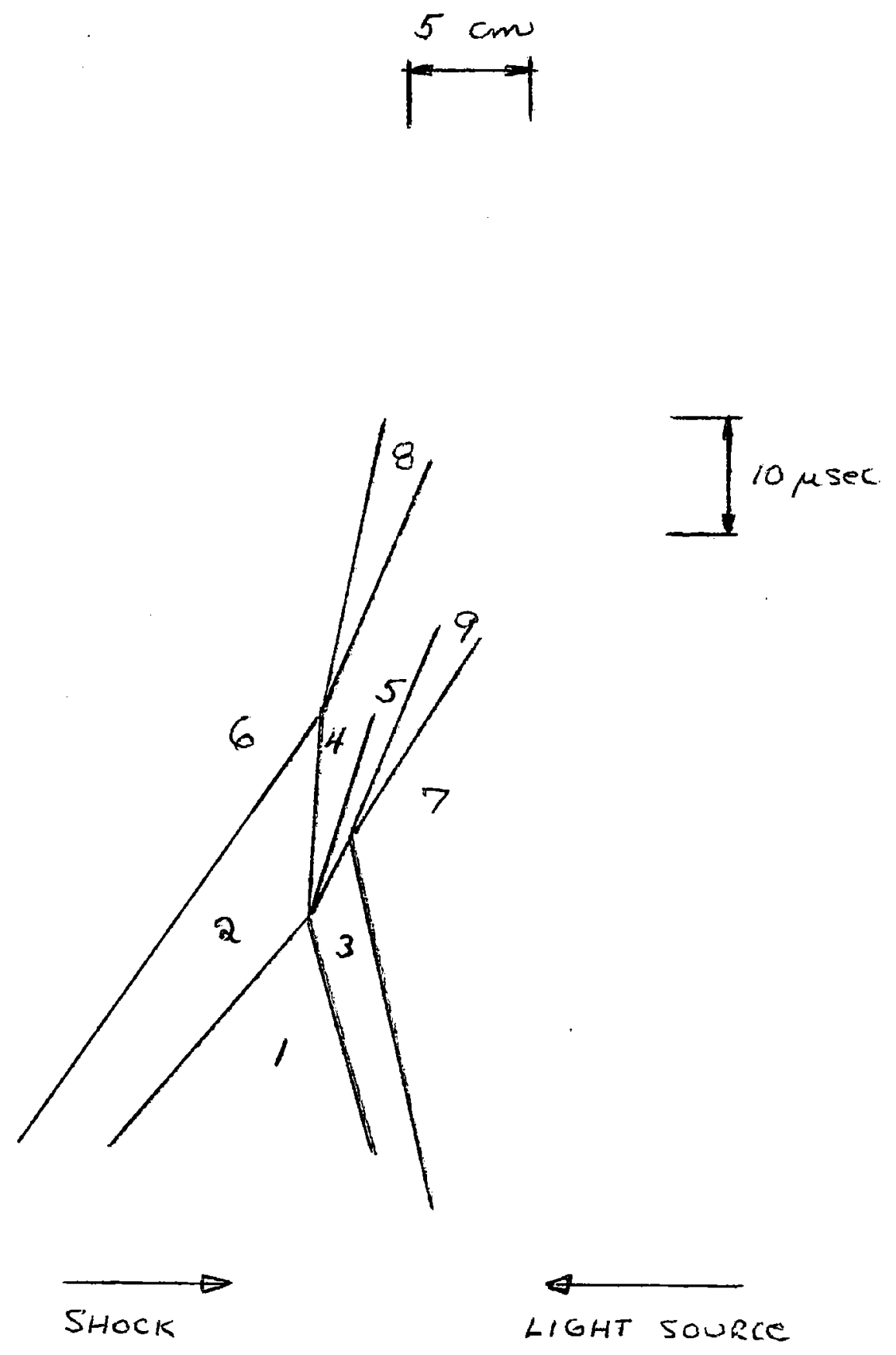

5is. 10 


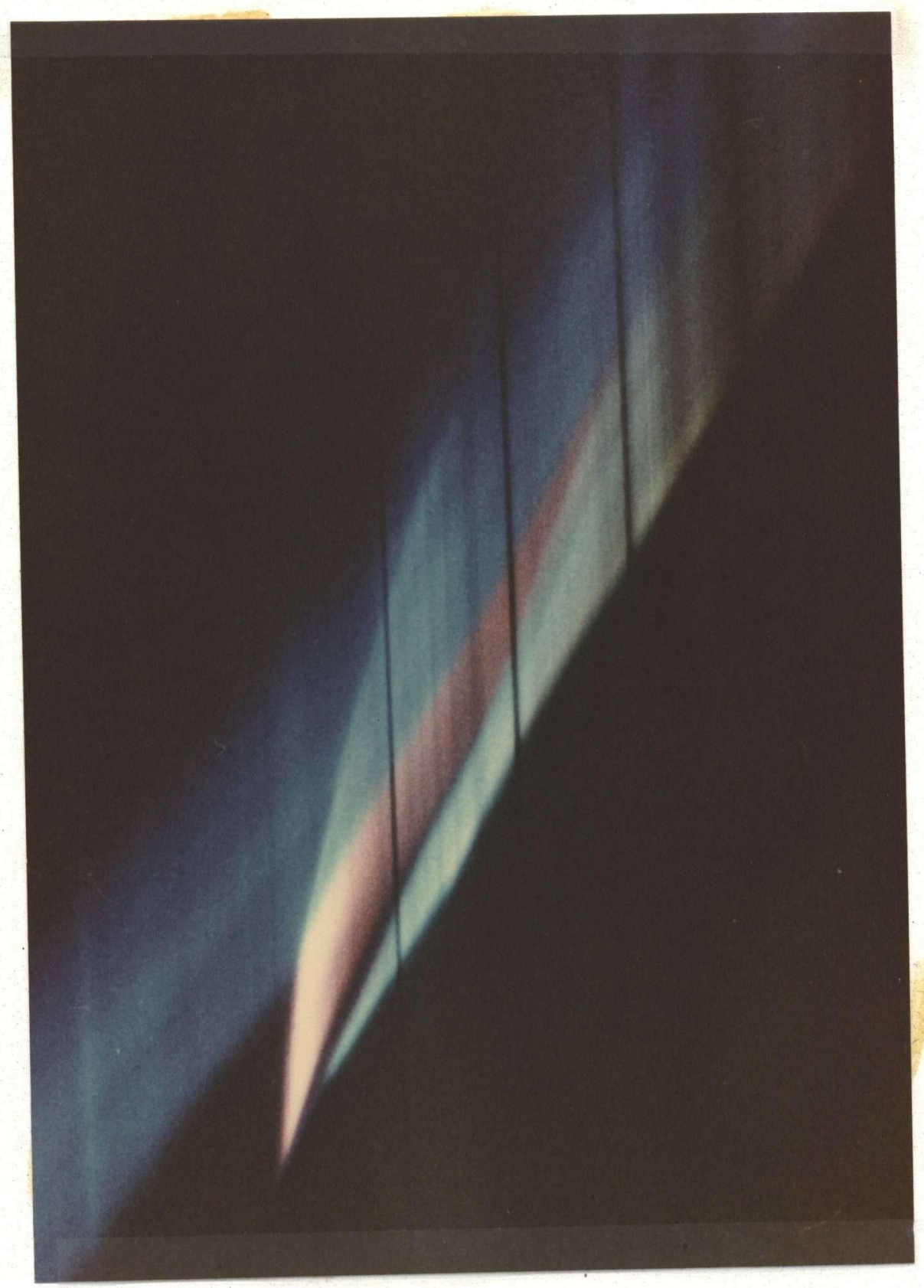

Fig. Il Shock-- Flow Field Intersection Enlarged 
$\langle 2,6\rangle$ and $\langle 3,7\rangle$ are contact surfaces separating the driver gas from the shock heated gas.

$$
\langle 6,8\rangle \text { and }\langle 7,9\rangle \text { are the refracted shocks which }
$$

are refracted on passing through the contact surfaces $\langle 2,6\rangle$ and $\langle 3,7\rangle$.

$\langle 4,8\rangle$ and $\langle 5,9\rangle$ are the refracted contact surfaces.

An experimental check of our results is possible in this case, since we can predict theoretically and measure independentIy the particle velocity in the doubly shock heated region.

\begin{tabular}{|c|r|}
\hline FRONT & \multicolumn{1}{|c|}{$\begin{array}{c}\text { VELOCITY } \\
\mathrm{km} / \mathrm{sec}\end{array}$} \\
\hline$\langle 1,2\rangle$ & $4.2 \pm 8 \%$ \\
$\langle 1,3\rangle$ & $2.4 \pm 10 \%$ \\
$\langle 2,4\rangle$ & $-0.604 \pm 10 \%$ \\
$\langle 3,5\rangle$ & $3.13 \pm 10 \%$ \\
$\langle 4,5\rangle$ & $2.2 \pm 10 \%$ \\
\hline
\end{tabular}

Firstly, consider the parameters immediately at the edge of the flow field by observing the change in velocity of the shock front at the boundary. The situation is the same as that in figure 5. The velocities of the four fronts, $\langle 1,2\rangle$, $\langle 1,3\rangle,\langle 2,4\rangle$, and $\langle 3,5\rangle$ are known; the initial parameters, $P_{1}, \rho_{1}, T_{1}, \mu_{1}$ are also known. The energy inputs $W_{3}$ and $W_{4}$ are unknown, and the desired information is $p_{3}, \rho_{3}$, $\mu_{3}$, and $T_{3}$. As the problem is stated, there are enough 
equations with the four sets of Rankine - Hugoniot equations plus $p_{4}=p_{5}$ and $\mu_{4}=\mu_{5}$. Unfortunately, there is a slight complication. In general, there will be an extra energy input term for state $S\left(W_{S}\right)$, and either an extra equation or an extra measurement must be used. The analytic solution to the above set of equations seems very difficult; therefore, numerical methods were employed (appendix B).

In applying the theory, we need one more equation since there are three unknow energy terms, $W_{3}, W_{4}$, and $W_{5}$. In our specific experiment, the energy absorbed in each state should depend upon the equilibrium properties of that state, and states 4 and 5 should be very similar; thus, we make the assumption that

$$
W_{4}=W_{5}
$$

Although this assumption is arbitrary, it does not seem too unreasonable.

With this assumption, the equilibrium parameters for the smear picture in figure 11 are calculated by the program in appendix $B$.

\begin{tabular}{|c|c|c|c|c|c|c|}
\hline STATE & $10^{-3} \mathrm{gm}^{3}$ & $\mathrm{~km} / \mathrm{sec}$ & $\begin{array}{c}\text { P } \\
\text { TORR }\end{array}$ & $\begin{array}{l}T \\
\circ K\end{array}$ & $g$ & $\begin{array}{l}W \\
10^{\prime \prime} \frac{\text { eng }}{\cos ^{2} \sec ^{2}}\end{array}$ \\
\hline 1 & 3.224 & 0.0 & $1.5 \pm 4 \%$ & 298 & 1.666 & 0.0 \\
\hline 2 & $19.8 \pm 10 \%$ & $3.518 \pm 10 \%$ & $399 \pm 10 \%$ & $10908 \pm 10 \%$ & 1.381 & 0.0 \\
\hline 3 & $7.47 \pm 30 \%$ & $1.364 \pm 30 \%$ & $81 \pm 30 \%$ & $6927 \pm 30 \%$ & 1.648 & 0.009 \\
\hline 4 & $37.7 \pm 30 \%$ & $2.1 \pm 10 \%$ & $950 \pm 40 \%$ & $13300 \pm 10 \%$ & 1.234 & 0.350 \\
\hline 5 & $32.7 \pm 30 \%$ & $2.1 \pm 10 \%$ & $950 \pm 40 \%$ & $14000 \pm 10 \%$ & 1.197 & 0.350 \\
\hline
\end{tabular}


The measured contact surface velocity $\langle 4,5\rangle$ should equal the calculated particle velocities $\mu_{4}$ and $\mu_{5}$. In this case,

$$
\begin{aligned}
& V_{\langle 4,5\rangle}=2.2 \pm 10 \% \mathrm{~km} / \mathrm{sec} \\
& \mu_{4}=\mu_{5}=0.1 \pm 10 \% \mathrm{~km} / \mathrm{sec}
\end{aligned}
$$

The agreement is well within the error bounds and thus provides a validity check on the methods followed in appendix $B$. According to the calculations, $W_{3}$ is very much smaller than $W_{4}$ and $W_{5}$. This fact is quite plausible since the ionization in state 3 is very low while that in states 4 and 5 1s quite high. The bound electrons in state 3 absorb only in specific wavelengths while the free electrons in states 4 and 5 absorb in any wavelength because of inverse bremstraul1ng. Also, notice that the velocity $\langle 4,8\rangle$ in figure 11 is not constant but rather it increases. This observation corresponds to an expected rarefraction wave caused by the high absorption in state 4 . All in all, figure 11 has provided a good check on the first part of the probing technique, and more generally, the theory in chapter two.

With this knowledge established, we then increased the energy into the light source and probed the resulting flow field at several positions down the tube (f1g. 12). The four velocities were measured for each collision and the parameters were obtained as outlined above. The results for many intersections were calculated and are plotted as a function of 
18.8

microsec
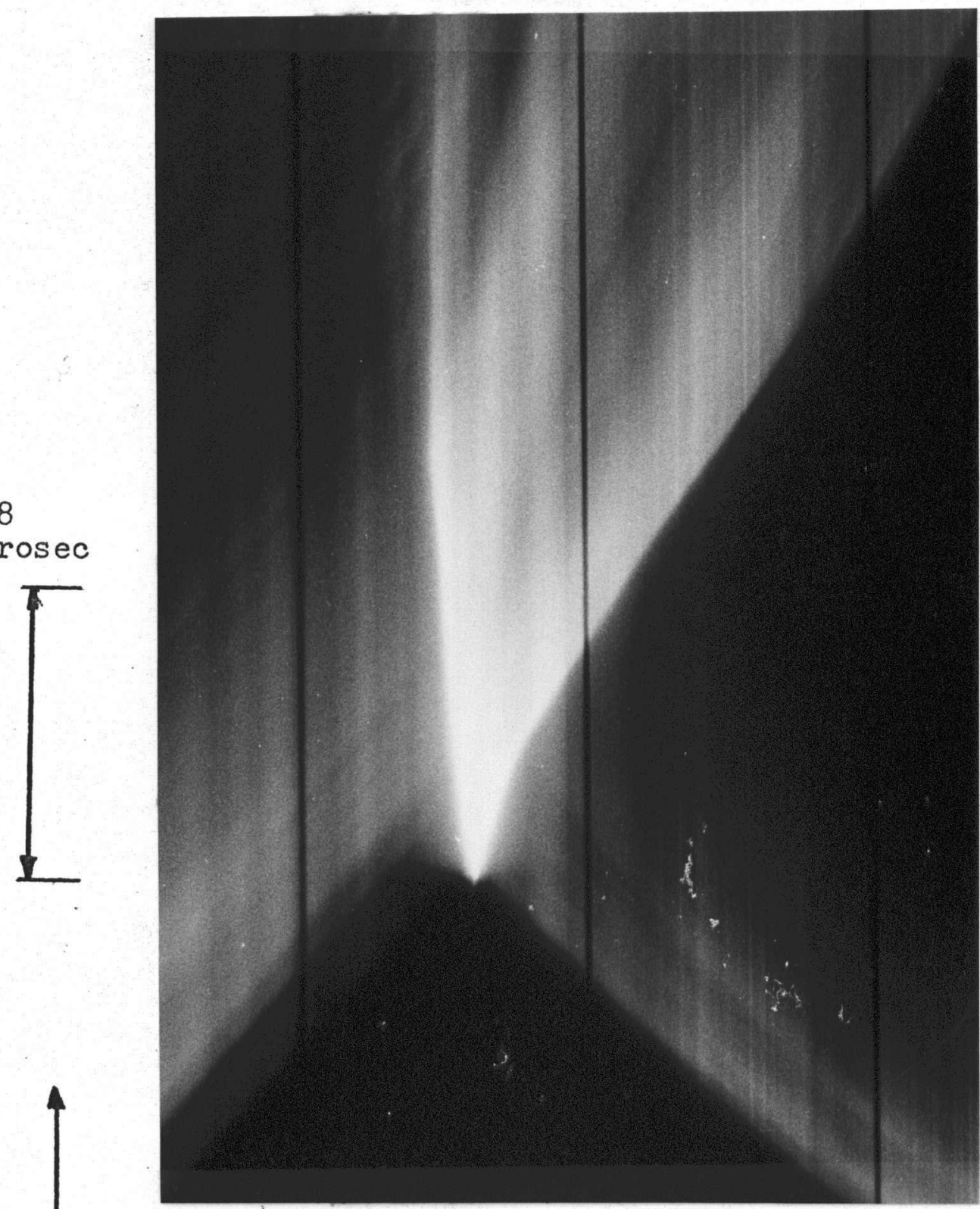

$\overrightarrow{D I S T A N C E}$

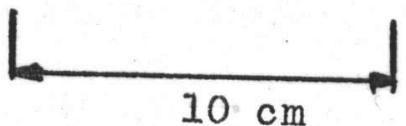

$\stackrel{\longrightarrow}{\text { HOCK WAVE }}$

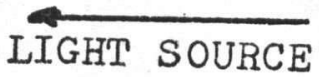

Fig. 12 Shock Wave Probing the Flow Field 
distance in figures 13 to 16.

The most interesting feature is that the absorbed energy, $W_{3}$, in each measurement is very close to the ChapmanJouguet energy,

$$
W=\frac{\rho_{1} v_{1}^{3}}{2\left(g_{2}^{2}-1\right)}
$$

and thus, implies that the flow field is at the ChapmanJouguet point.

The only efficient way of transmitting energy of this amount right into the front is by radiative absorption. We therefore identify this unknown wave as a Chapman-Jouguet-type radiation front. As a further check on the validity of this fact, the initial density $S_{1}$ was varied and the front velocity was measured at fixed distances (fig. 17). The points fall reasonably well on the expected proportionality (Ahlborn and Zuzak, 1969)

$$
V \propto s_{1}^{-1 / 3} \text {. }
$$

Since the particles leave a Chapman-Jouguet front with sonic velocity, a rarefraction wave is expected to follow this front. This means that the pressure, density, and particle velocity are expected to decay behind the front. It is checked by applying the second part of our shock probe analysis. We will show that the results are self-consistent and agree with independent piezo probe measurements. 


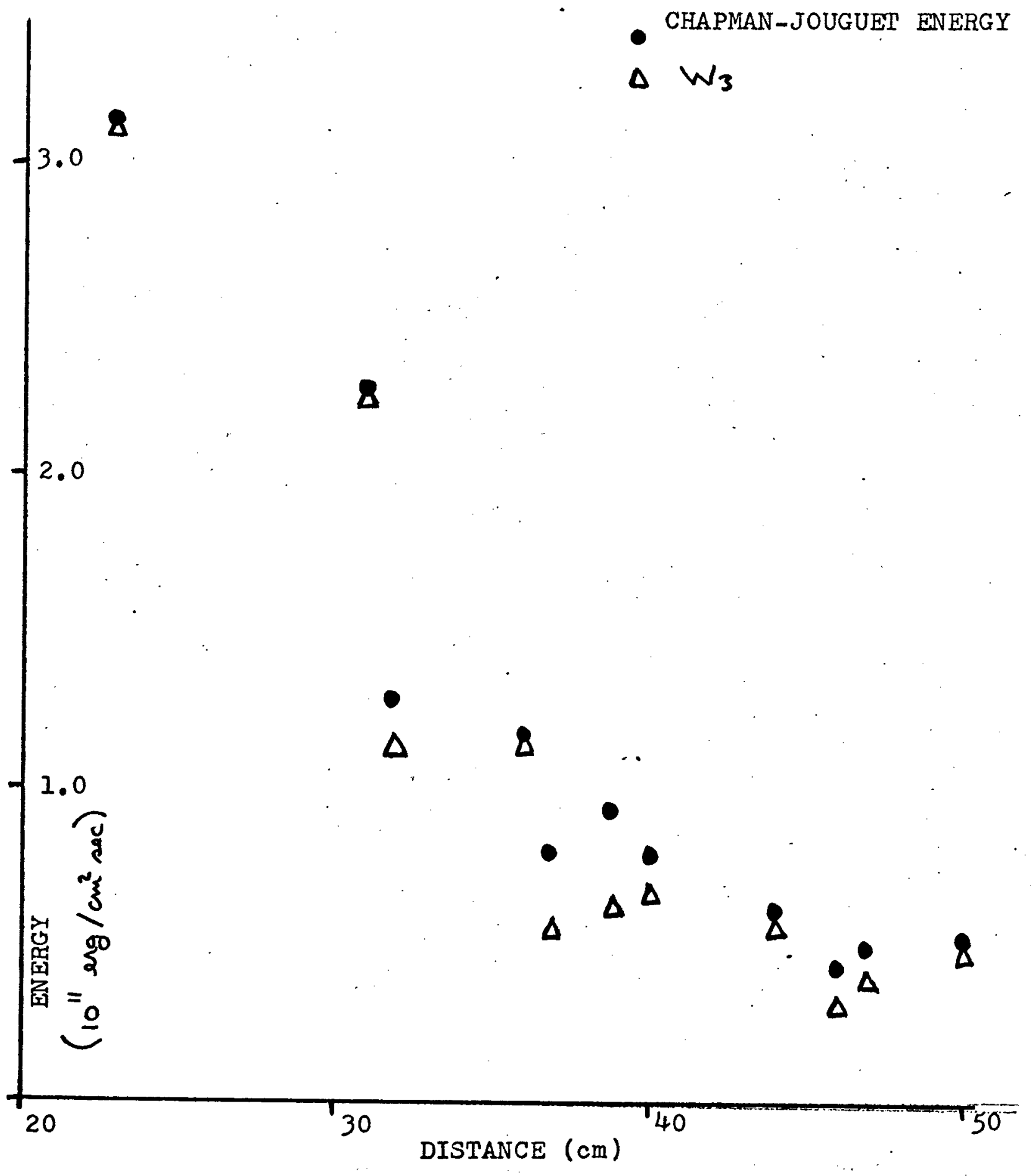

F18. $15 W_{3}$ VS Distance 
$-38-$

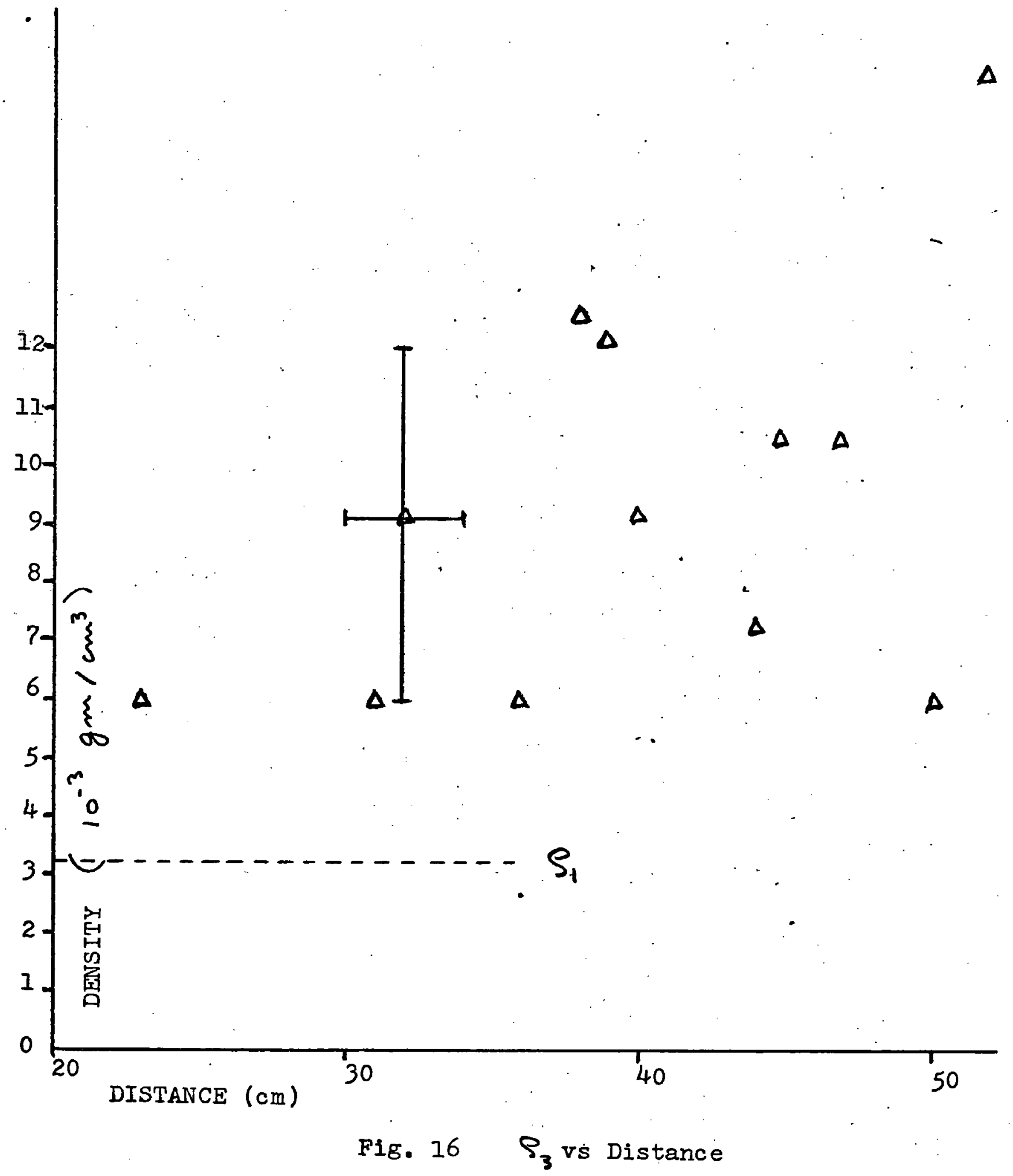




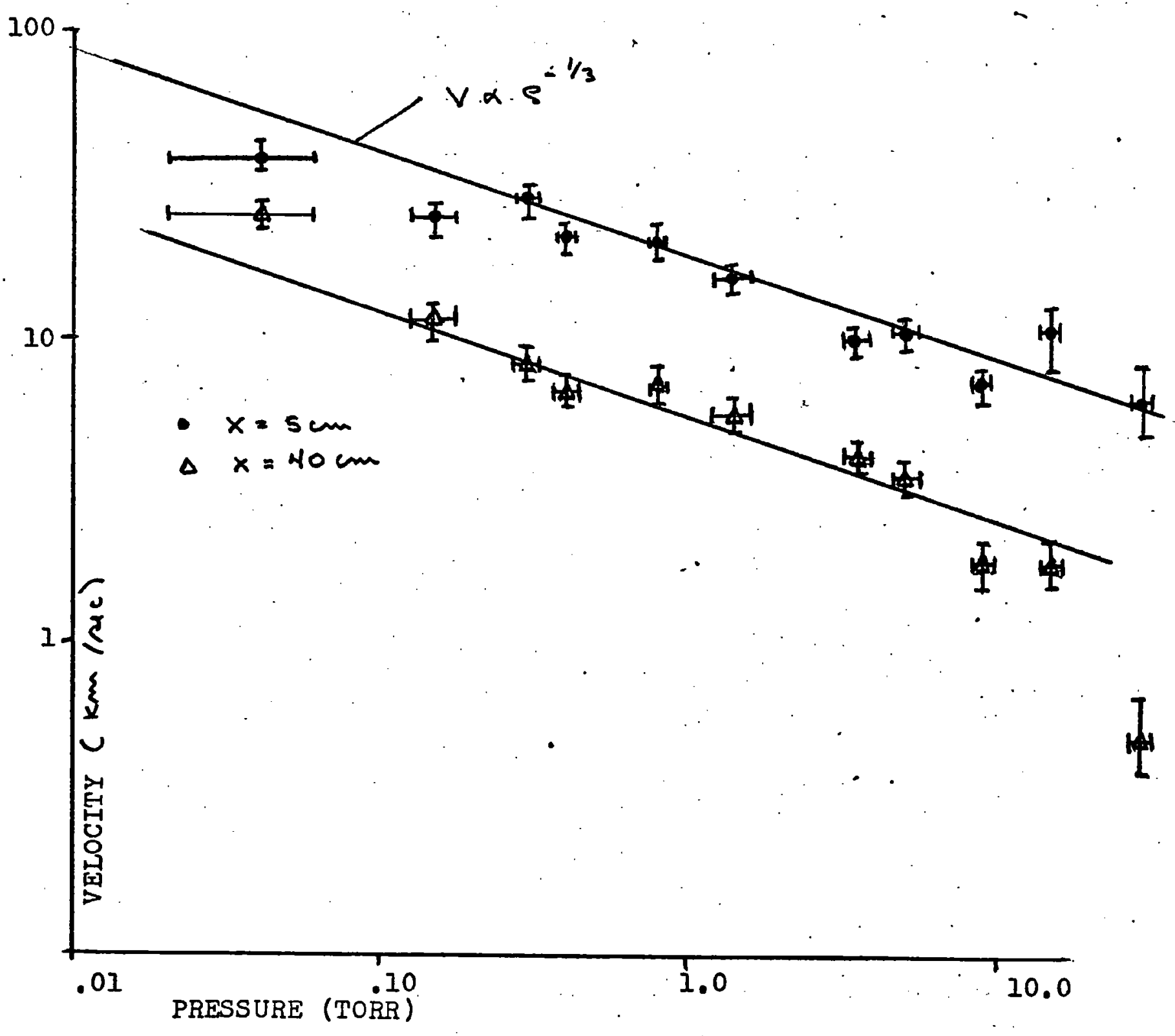

F18. 17 Radiation Front Velocity vs Downstream Pressure 


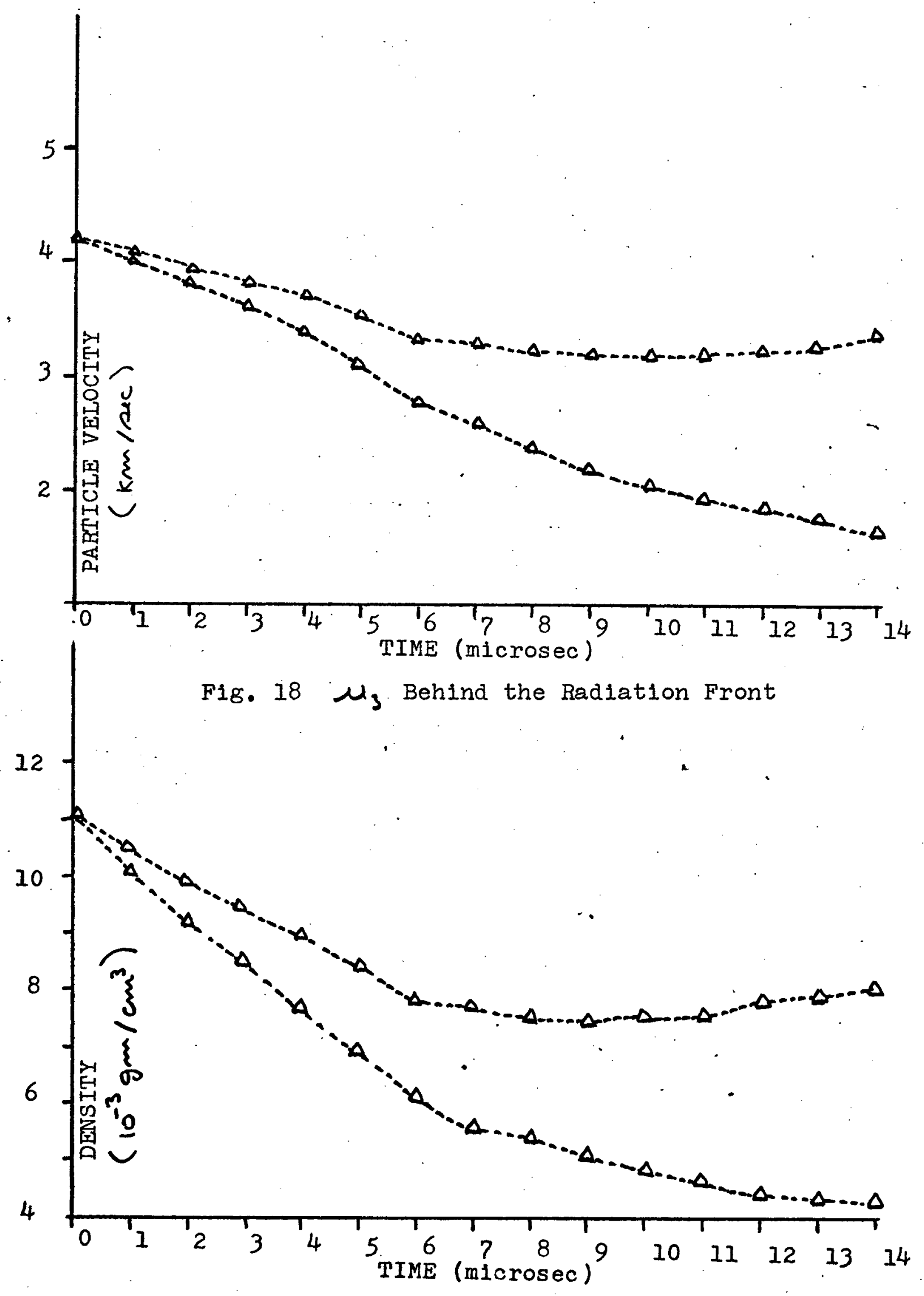

F1g. $19 \rho_{3}$ Behind the Radiation Front 


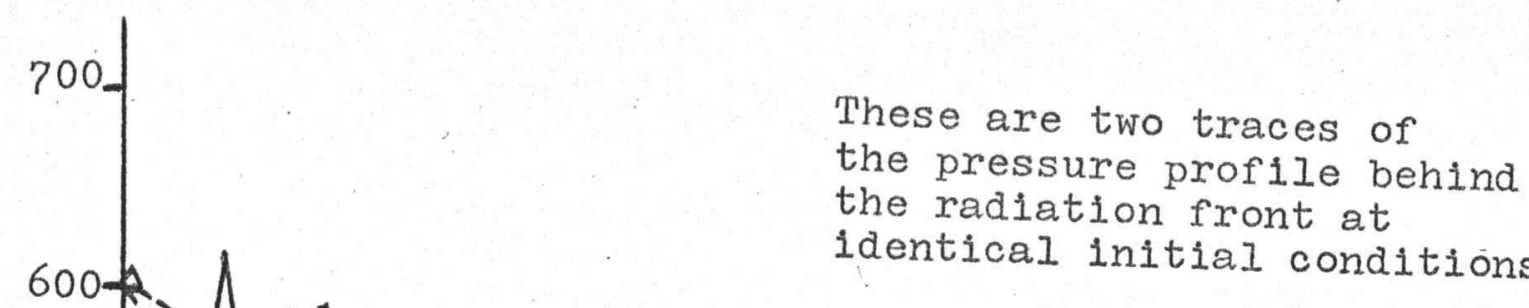

These are two traces of

the pressure profile behind

the radiation front at

identical initial conditions
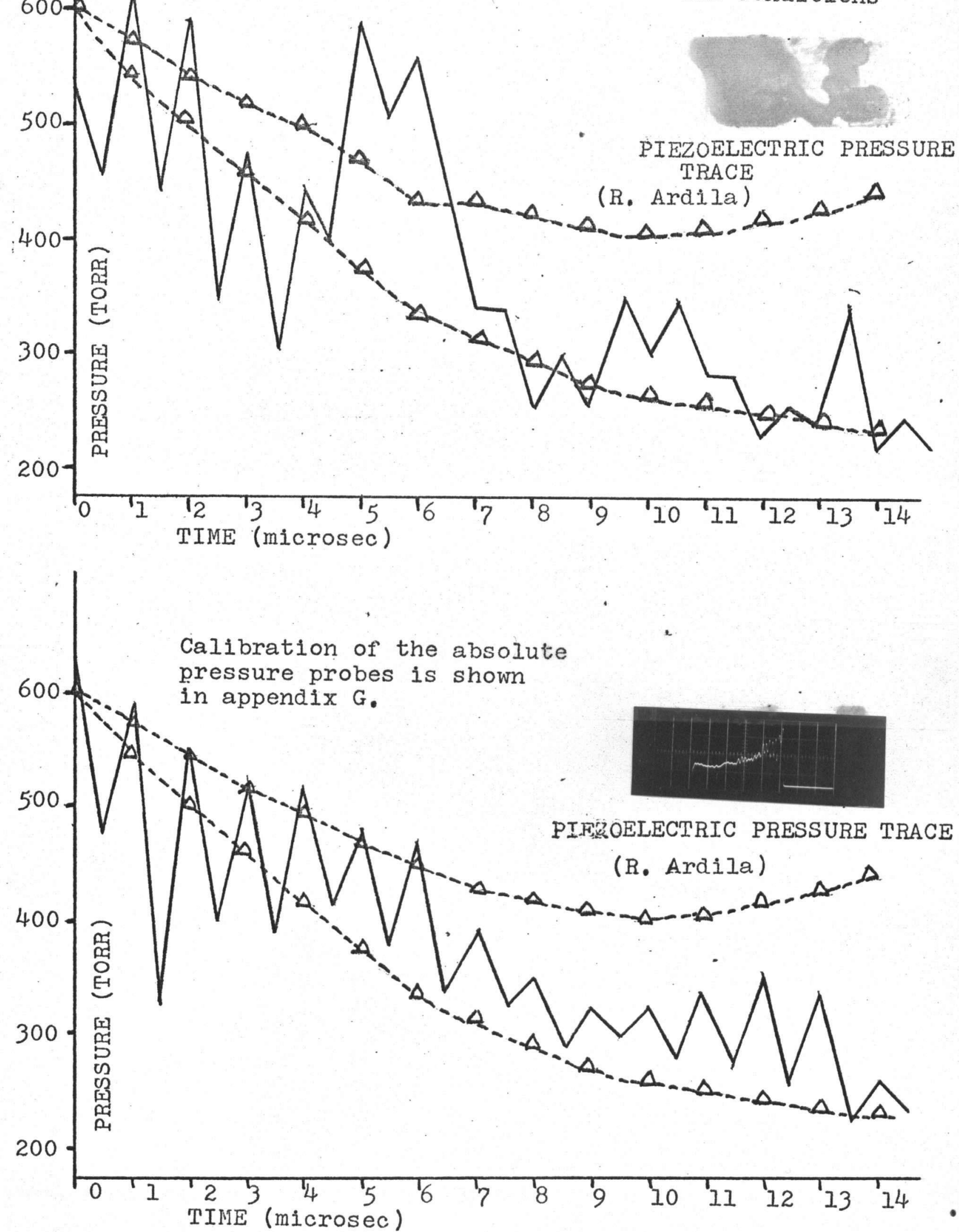

F18.20 and 21 Pressure Behind the Radiation Front 
$-42-$

To apply the second part of the probing technique, we measured the shock velocity as a function of time through the continuously varying region behind the radiation front. We chose intersections which are approximately $35 \mathrm{~cm}$ from the light source.

From chapter three, we have equation (54),

$$
V(x)=V(0)+\Delta \mu_{1}(x)+\int_{x=0}^{x}\left(f d \rho_{1}+h d p_{1}\right)
$$

in which the integrals are extremely difficult to perform. Therefore, the solution must again come numerically, using a simple predictor numerical integration formula, such as

$$
\int_{0}^{1} f(x) d x=f(0)
$$

and obtaining

$$
\Delta V=\Delta \mu_{1}+f \Delta S_{1}+h \Delta p_{1}
$$

There are three unknowns-- $\Delta \mu_{1} \cdot \Delta \rho_{1}$, and $\Delta p_{1}$; therefore three equations are required. Consequently, we must probe the flow field with three different shock velocities and obtain the set of equations,

$$
\begin{aligned}
& \Delta V_{\alpha}=\Delta \mu_{1}+f_{\alpha} \Delta S_{1}+h_{\alpha} \Delta p_{1} \\
& \Delta V_{\beta}=\Delta \mu_{1}+f_{\beta} \Delta S_{1}+h_{\beta} \Delta p_{1} \\
& \Delta V_{\gamma}=\Delta \mu_{1}+f_{\gamma} \Delta g_{1}+h_{\gamma} \Delta p_{1}
\end{aligned}
$$


These equations are then inverted, to complete the solution (appendix C).

The exact solution of the equations developed in chapter three is highly unstable, but it is relatively easy to obtain Iimits on the variation of the plasma parameters throughout the flow. The density, particle velocity, and pressure profile are plotted against time in figures 18 to 21 . The pressure profile is compared with some absolute piezoelectric pressure probe measurements. These quantitative pressure measurements* provide a completely independent check on the results. The profiles agree both in shape and in magnitude, and are of the type expected behind radiation fronts at the Chapman-Jouguet point (Zuzak, 1968). The pressure results are conclusive evidence that the flow field is a radiation front and not a blast wave. If the flow fleld were a blast wave, the pressure at the edge would be governed by the Rankine-Hugoniot equations, in which case the pressure would be the same as the equilibrium pressure behind a shock wave travelling at the same velocity. However, the pressure found by both the shock and pressure probes is one-half that of the shock pressure (ie. precisely the pressure expected behind a radiation front travelling at the Chapman-Jouguet point).

* Measurements were carried out by R. Ardila. 


\section{Chapter 5}

CONCLUSIONS

The inevitable result of this thesis is that we have raised more questions than we have answered. One of the answered questions is that concerning the propagation of a shock wave into an inhomogeneous and moving media. An extension of the equations involved has been the development of the shock wave as a tool for the probing of unknown flow fields. In our experimental application we have probed a flow field produced by a Bogen light source. We have found our shock probes to be useful and accurate. We have discovered the flow field to be a radiation front at the Chapman-Jouguet point.

The questions raised include those in understanding the behaviour of the radiation front. More explicitly, astrophysicists feel that weak R-type radiation fronts do exist. Weak R-type fronts have energy inputs greater than the ChapmanJouguet energy. But, so far we have not observed any energy inputs greater than the Chapman-Jouguet energy. We also believe that the radiation front at lower initial pressures may be quite interesting (fig. 22), but before any investigation, the operation of the shock tube must be improved so that we can be certain of its properties at these low pressures. We would also prefer a more reproducible light source.

We are curious as to why the energy input is maintained even after the light source has stopped radiating. We suppose 
$\rightarrow-5 \mathrm{~cm}$
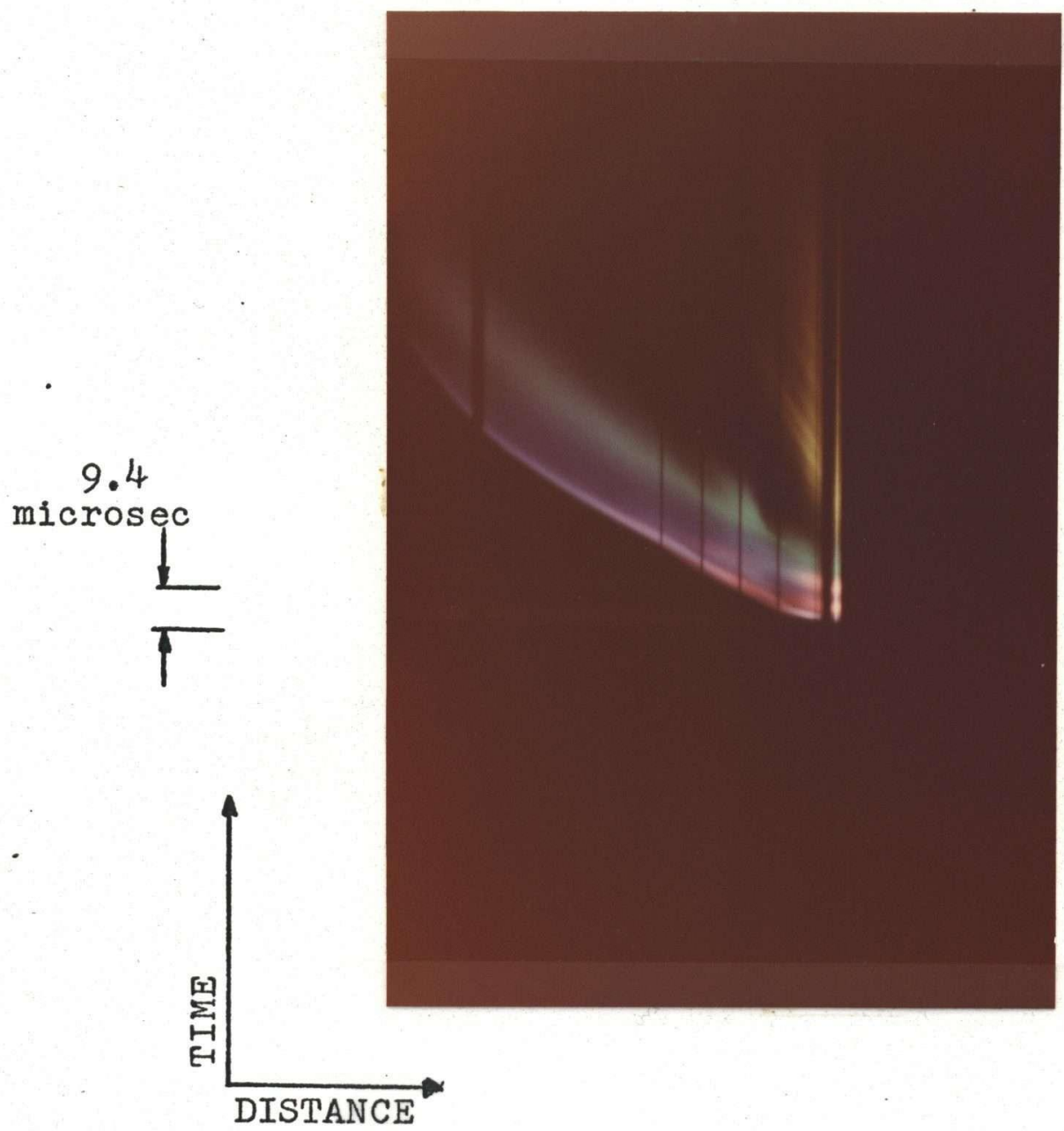

$p_{1}=0.5$ TORR Ar

Fig. 22 Radiation Front at Low Downstream Pressure 
that the energy diffuses from the light source through the gas. Indeed, a spectroscopic study (perhaps time resolved) should be enlightening.

Another feature is the similarity between the radiation front which we observe and the flow field produced by a standard electromagnetic T-tube. Perhaps the T-tube flow field is not a blast wave but rather a radiation front. Perhaps, too, radiation fronts are quite common but unrecognized laboratory occurrences.

Finally, we have become quite excited about applying our probe technique to other unknown flow fields, such as laser sparks. 


\section{BIBLIOGRAPHY}

1. Ahlborn, B., IPP 3/12 (1963)

2. Ahlborn, B., Salvat, M., IPP 3/2 (1962)

3. Ahlborn, B., Salvat, M., Z. Naturforschung 22a, 260 (1967)

4. Ahlborn, B., Zuzak, W., Rev. Sci. Instr. 38, 194 (1967)

5. Ahlborn, B., Zuzak, W., Can. J. Phys. 47, 4709 (1969)

6. Ardila, R., M.Sc. Thesis U.B.C. (to be published)

7. Axford, W.I., DeYoung, D.S., Il Nuovo. Cimento 50B, 199 (1967)

8. Barach, J.P., Vermilition, R.E., Phys. Fluids $\underline{8}, 2976$ (1965)

9. Chisnell, R.F., Proc, Roy, Soc, A232, 350 (1955)

10. Chu, B.T., NACA Tech. Note 3411 (1955)

11. Driver, H., Ph.D. Thesis U.B.C. (1963)

12. Driver, H., Smy, P.R., J. Fluid Mech. 17, 182 (1963)

13. Driver, H., Smy, P.R., Proc. 6th Int. Conf. Ionization Phen. in Gases Vol.4, 319, Paris (1963)

14. Frank-Kamenetsk11, D.A., Nadezh1n, D.K., Soviet Astronomy 2. 226 (1965)

15. Gaydon, A.G., Hurle, I.R., "The Shock Tube in High Temperature Chemical Physics", Chapman Hall (1963)

16. Huni, J.P., Ph.D. Thesis U.B.C. (to be published)

17. Muntenbruch, H., Phys. Fluids Suppl. I-1l (1969)

18. Offenburger, A., M.Sc. Thes is U.B.C. (1962)

19. Ohyama, N., Prog. of Theoretical Physics 26, 251 (1961)

20. Ôno, Y., Sakashita, S., Yamazak1, H., Prog, of Theoretical Phys. 23, 294 (1958) 
21. Phillips, M.G.R., Ph.D. Thesis U.B.C. (1968)

22. Rehm, R.G., Phys. Fluids 11, 1872 (1968)

23. Roshko, A., Phys. Fluids 2, 835 (1960)

24. Shapiro, A.H., "The Dynamics and Thermodynamics of

Compressible Fluids Flow II", Ronald Press Co. (1954)

25. Smy P.R.; Nature 193, 969 (1962)

26. Smy, P.R., Rev. Sc1. Instr. 26, 1334 (1965)

27. Smy, P.R., Stonebridge, D., Turmer, J.H., J. Sci. Instr. 39, 309 (1962)

28. Taub, A.H., Phys. Rev. 72, 51 (1947)

29. Whitham, G.B., J. Fluid Mech. 4, 337 (1958)

30. Williamson, J.H., Brit. J. Appl. Phys. 18, 317 (1967)

31. Zuzak, W., Ph.D. Thesis U.B.C. (1968)

32. Zuzak, W., Ahlborn, B., Can. J. Phys. (to be published) 
Appendix A

THE DOUBLY REFLECTED WAVE

In deriving the partial derivatives, $\left(\frac{\partial V}{\partial \rho_{1}}\right)_{p_{1}, \mu_{1}}$ and $\left(\frac{\partial V}{\partial p_{1}}\right)_{\rho_{1}}, \mu_{1}$ : the analysis was considered only to the first order. Chisnell (1960) also considered the effect of the wave reflected by the variable medium when the singly reflected wave passes back through it. The doubly reflected wave moves in the same direction as the incident shock and is an expansion wave.

The physical situation corresponding to the partial derivatives now becomes that in figure $A$. The following phenomena need to be considered,

1. reflection by the medium of an element of the singly reflected wave forming an element of the doubly reflected wave

2. the interaction of the elements of the singly and doubly reflected waves.

3. the variation of the strength of an element of the doubly reflected wave as it travels through the variable region

4. the catching up of the incident shock by the expansion wave. 


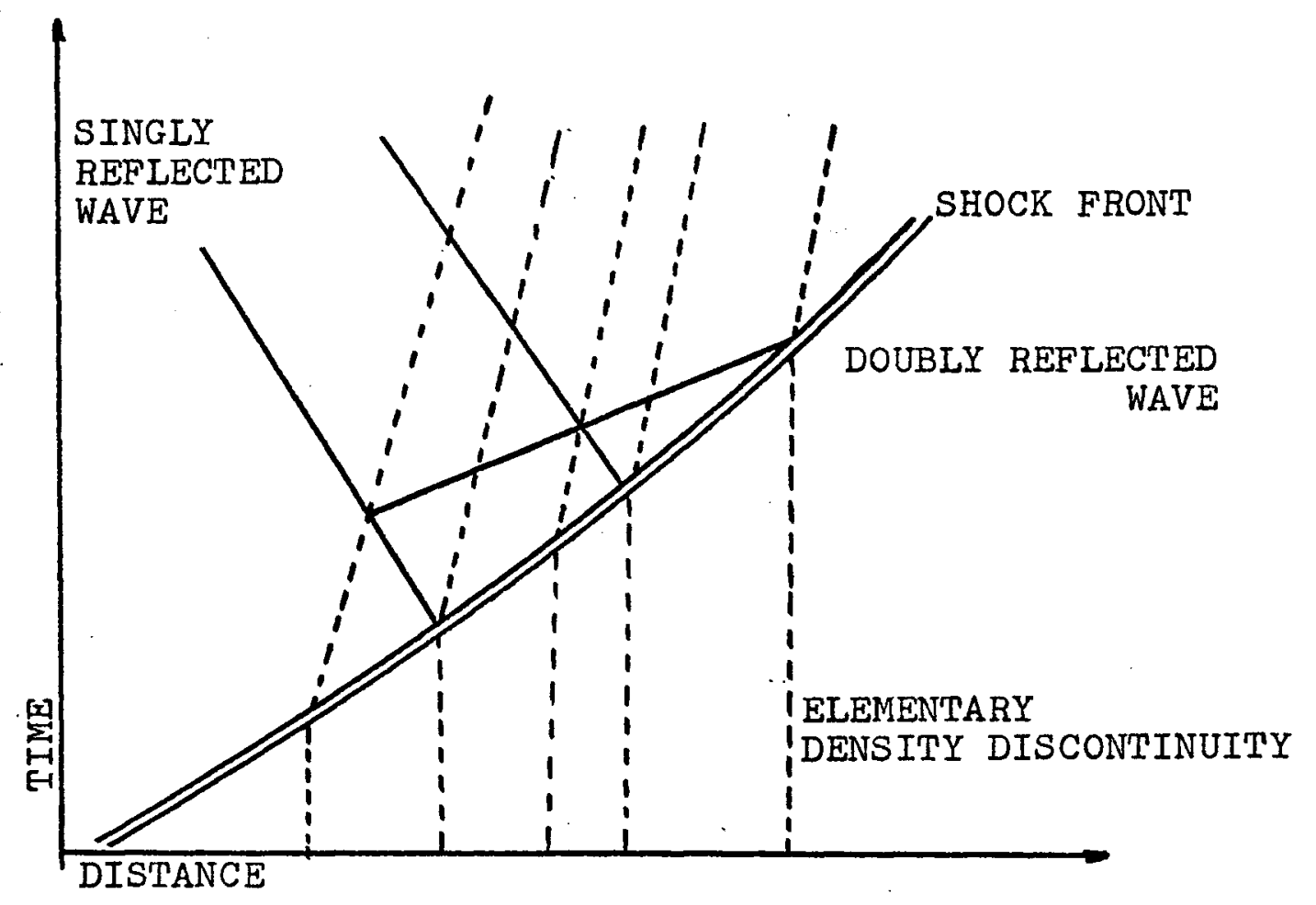

Fig. A Doubly Reflected Wave

Chisnell checked his derivation of

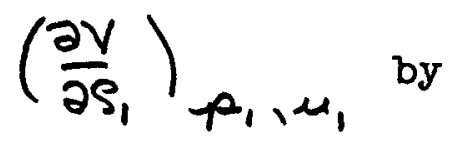
comparing the theory for shock wave-contact surface interactions with his results (applying both single reflection and correctIons due to double reflection). He found good agreement for the single reflection theory whenever the reflected disturbance was small. With application of the double reflection theory, the agreement was always excellent even when the strength of the reflected wave was large. In other words, the single reflection analysis used in chapter three will likely break down if the gradients of initial pressure or density are very large. 
Ohyama (1961) extended the method of Ono, Sakashita, and Yamazaki (1960) (which in turn is just the Chisnell method with single reflection) to the case of doubly reflected waves, by taking the effects of the the second-order waves into consideration. He did not investigate the behaviour of weak shocks, but did find that the rate of growth of shock strength obtained by the first approximation is little influenced, as far as the strong shock limit is concerned.

We can conclude that the analysis in chapter three will hold if either of the following conditions is met:

1. the incident shock is strong, or

2. the density and pressure gradients are not too strong. 
Appendix B

PARAMETERS AT THE BOUNDARY

The numerical methods used are quite straightforward. " The equilibrium properties of the four states, $2,3,4$, and 5 (chapter 2) are calculated 1teratively. Each state is calculated from three subroutines. Subroutine PEA2 calculates $\rho, p$, and $v$ using the solutions of the RankineHugoniot equations (18) and (19). Subroutine TEA2 calculates

$T$ using the equilibrium equation of state,

$$
p=\left(1+\alpha_{1}\right) \rho R T
$$

where $\alpha_{1}$ is the degree of first ionization;

and

$$
\begin{aligned}
H & =\frac{g}{g-1} \frac{p}{\rho} \\
& =\frac{5}{2} \frac{p}{\rho}+\alpha_{1} \frac{I_{1}}{m_{A}}
\end{aligned}
$$

where $I_{1}$ is the ionization potential and $m_{A}$ is the atomic mass. Subroutine GEE2 calculates $g$ essentially by reproducing figure 2 .

The energy input terms, $W_{3}, W_{4}$, and $W_{5}$ are used as parameters and the resulting sets of solutions are shown in figure $B-1$. When $W$ becomes very large, the iterative procedure often breaks down because the square root in equations (18) and (19) will sometimes become negative. Therefore, instead of using $W$ as the parameter, it is more convenient to use the 
entire root as the parameter.

The desired value of $W_{3}$ is obtained graphically by inserting the conditions,

$$
\begin{aligned}
& w_{4}=w_{5} \\
& \mu_{4}=u_{5} \\
& p_{4}=p_{5}
\end{aligned}
$$

Figure B-I indicates the solution for the smear picture in figure 10.

One important feature of the solution is that the condition $P_{4}=P_{5}$ is actually found by varying only $W_{3}$. Small values of $W_{3}$ have a large effect on $p_{5}$, while large values of $W_{4}$ and $W_{5}$ have very little effect on $p_{4}$ and $p_{5}$ respectively. Therefore, since we are interested in finding only $W_{3}$, we can remove the arbitrary assumption that $W_{4}=W_{5}$, and vary only $W_{3}$ in order to fulfill the condition,

$$
p_{4}=p_{5}
$$

We used this condition to obtain the remaining results in chapter five.

Figure B-2 indicates the type of errors resulting in $p_{4}$ and $\mu_{4}$ due to errors in measuring $V_{\langle 2,4\rangle}$ and $V_{\langle 1,2\rangle}$. Again, an error in $V_{\langle 1,2\rangle}$ will cause quite a noticeable change in py. 


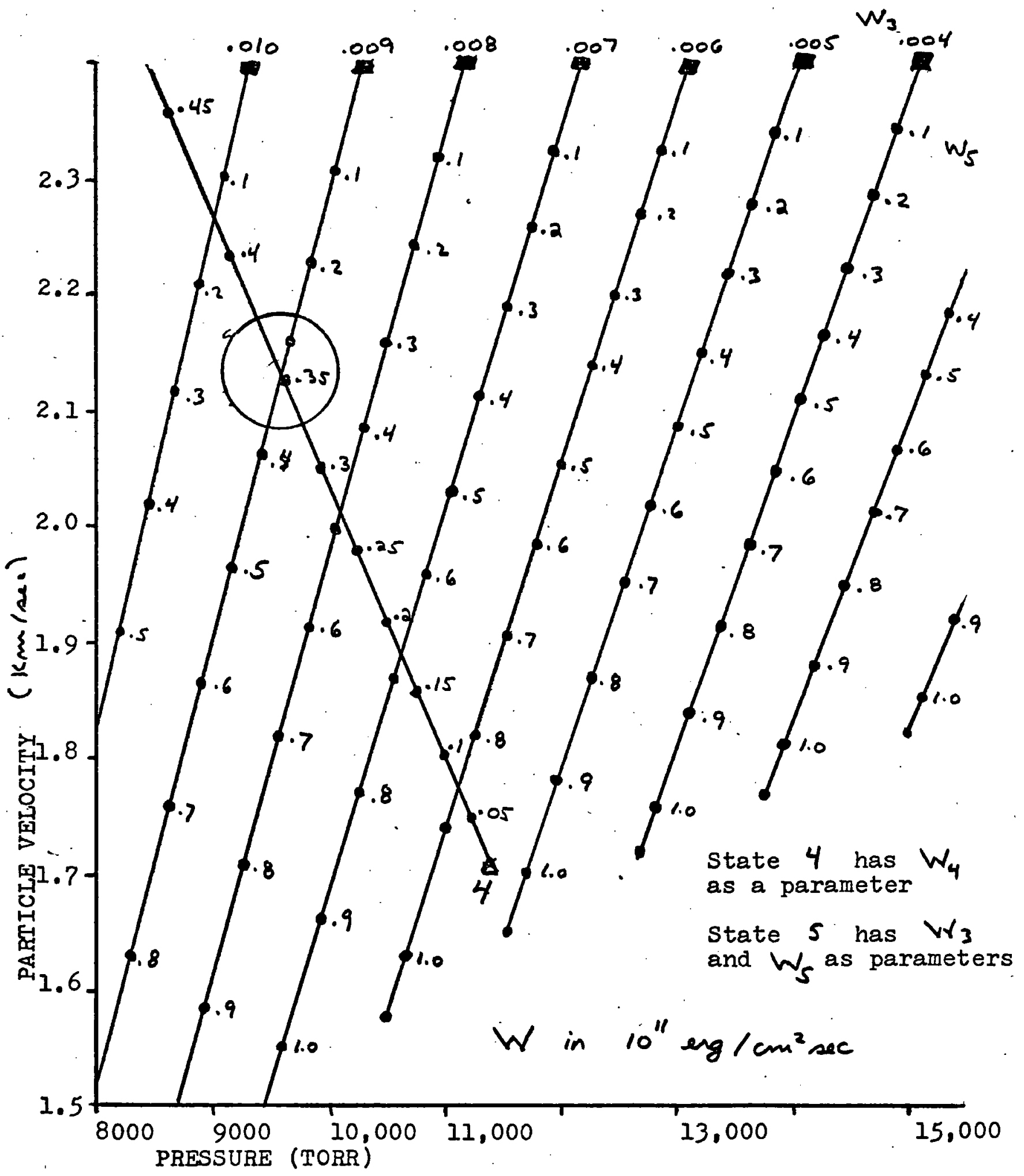

Fig. B-I Graphical Solution for Intersection Problem 


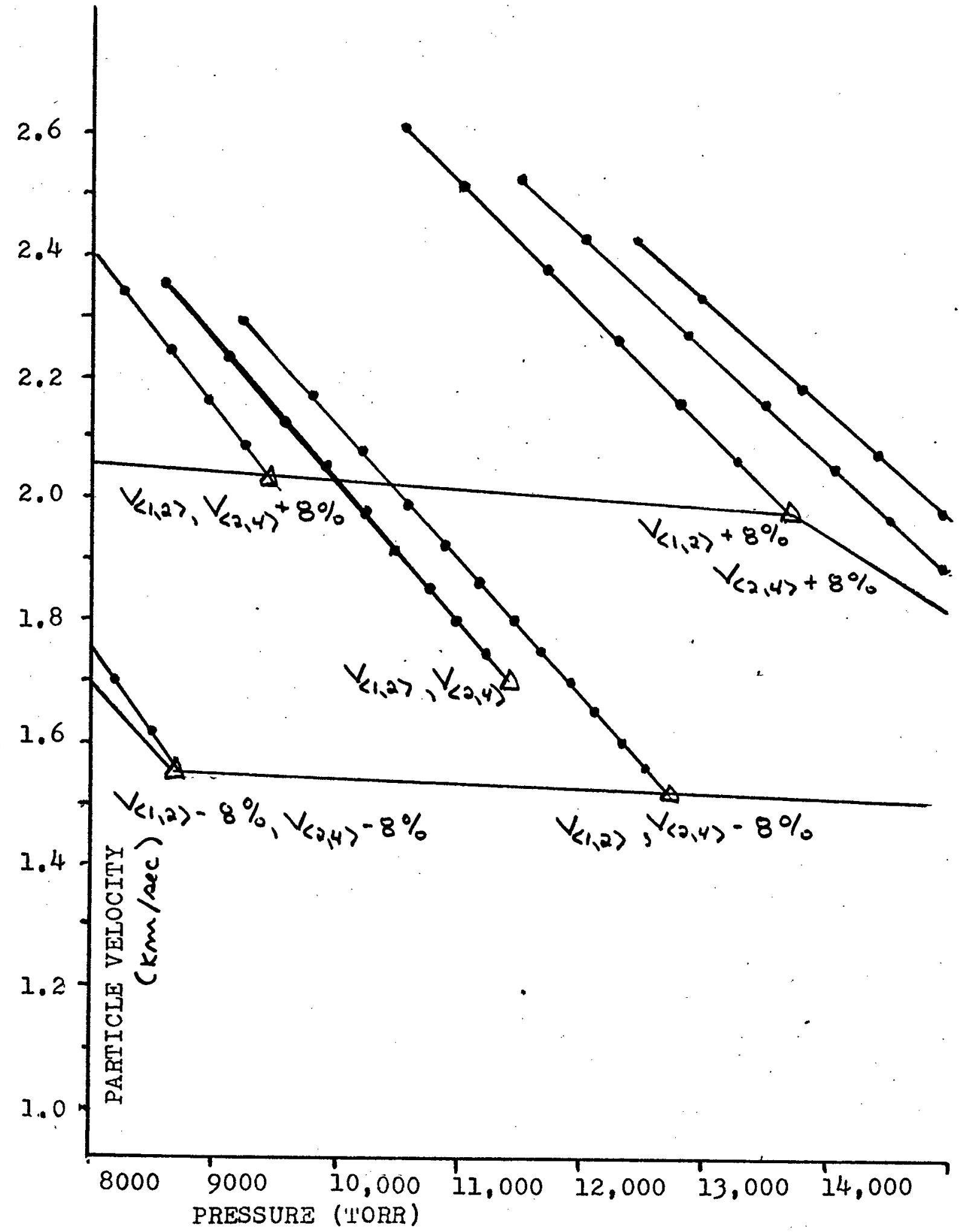

F18. B-2 Error Bounds on State 4 
38

100

FORMAT $(3 F 15.8)$

FORMAT $(5 \mathrm{X}, 2 \mathrm{HUl}, 9 \mathrm{X}, 2 \mathrm{HP} 1,10 \mathrm{X}, 2 \mathrm{HT} 1,10 \mathrm{X}, 2 \mathrm{HG} 1,9 \mathrm{X}, 2 \mathrm{HD} 1,11 \mathrm{X}, 2 \mathrm{HW} 1)$

FORMAT $(5 \mathrm{X}, 2 \mathrm{HU} 2,9 \mathrm{X}, 2 \mathrm{HP} 2,10 \mathrm{X}, 2 \mathrm{HT} 2,10 \mathrm{X}, 2 \mathrm{HG} 2,9 \mathrm{X}, 2 \mathrm{HD} 2,11 \mathrm{X}, 2 \mathrm{HW})$

FORMAT $(5 \mathrm{X}, 2 \mathrm{HU} 3,9 \mathrm{X}, 2 \mathrm{HP} 3,10 \mathrm{X}, 2 \mathrm{HT} 3,10 \mathrm{X}, 2 \mathrm{HG} 3,9 \mathrm{X}, 2 \mathrm{HD} 3,11 \mathrm{X}, 2 \mathrm{HW} 3)$

FORMAT $(5 \mathrm{X}, 2 \mathrm{HU} 4,9 \mathrm{X}, 2 \mathrm{HP} 4,10 \mathrm{X}, 2 \mathrm{HT} 4,10 \mathrm{X}, 2 \mathrm{HG} 4,9 \mathrm{X}, 2 \mathrm{HD} 4,11 \mathrm{X}, 2 \mathrm{HW} 4)$

FORMAT $(5 X, 2 H U 5,9 X, 2 H P 5,10 X, 2 H T 5,10 X, 2 H G 5,9 X, 2 H D 5,11 X, 2 H W 5)$

FORMAT $(5 X, 4 \mathrm{~F} 12.3)$

FORMAT ( $11 \mathrm{X}, 4 \mathrm{HVS} 12,8 \mathrm{X}, 4 \mathrm{HVS} 13,8 \mathrm{X}, 4 \mathrm{HVS} 24,8 \mathrm{X}, 4 \mathrm{HVS} 35)$

FORMAT (4F6.3)

FORMAT $(5 X, 5$ HALF $=, 5 X, F 8.1)$

FORMAT $(5 X, F 6.3,5 X, F 9.1,5 X, F 9.1,5 X, F 6.3,5 X, F 8.6,5 X, F 10.6)$.

FORMAT $(5 X, 6 H A L F 2=, 5 X, F 8.2)$

FORMAT $(5 X, 6$ HALF3 $=, 5 X, F 8.2)$

READ $(5,1) W 3 U, W 4 U, W 5 U$

READ $(5,1) W 3 L, W 4 L, W 5 L$

READ $(5,1) W 3 M, W 4 M, W 5 M$

READ $(5,9) \cup S 12, V S 13, V S 24, V S 35$

WRITE $(6,8)$

WRITE $(6,7)$ VS 12, VS 13, VS 24, VS 35

$W 3=W 3 U$

$D 1=0.00322428$

$G 1=1.666$

$W 1=0.0$

$u_{1}=0.0$

$T_{1}=298.0$

$P_{1}=1.500$

WRITE $(6,2)$

WRITE $(6,11) \cup 1, P 1, T 1, G 1, D 1, W 1$

$P 1=133.3 * P 1$

$\mathrm{G2}=\mathrm{G1}$

$V 1=V S 12-U 1$

SHM $=V 1 / 0.321$

$W=0.0$

CALL PEA2 (P1,D1,G1,G2,W,V1,SHM, DR, D2,P 2,V2)

CALL TEA2 (P2,D2,G2,T2,ALF, ALF2,ALF3,ALF 4, ALF5)

CALL GEE2 $(T 2, P 2, G Z)$

$G 2=(G Z+3.0 * G 2) / 4.0$

IF $(A B S(G 2-G Z) . G T .0 .0001)$ GO TO 100

$P 2=P 2 / 133.3$

WRITE $(6,3)$

$\mathrm{U}_{2}=\mathrm{VS} 12-\mathrm{V} 2$

$W 2=0.0$

WR ITE $(6,11) \cup 2, P 2, T 2, G 2, D 2, W 2$

WRITE $(6,10)$ ALF

WRITE $(6,12)$ ALF2

$D X=D 2$

$G X=G 2$

$U X=U 2$ 


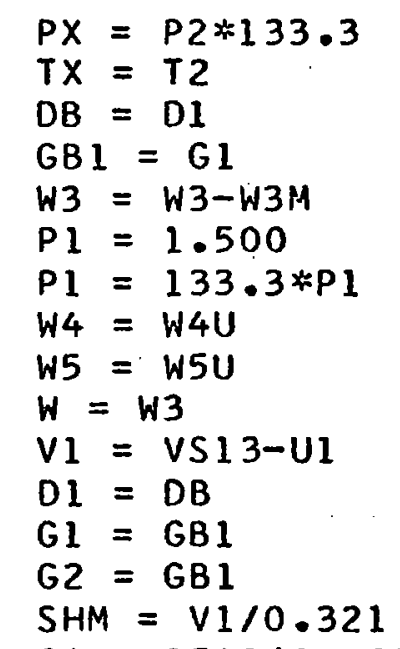

200 CALL PEA3(P1,D),Gl,G2,W,V1,SHM, DR,D2,P 2,V 2$)$

CALL TEA2 (P2, D2, G2,T2,ALF, ALF2,ALF3,ALF4,ALF5)

CALL GEE2 $(T 2, P 2, G Z)$

$G 2=(G Z+3.0 * G 2) / 4.0$

IF $(A B S(G 2-G Z) \cdot G T .0 .0001)$ GO TO 200

WRITE $(6,4)$

$\mathrm{U}_{3}=\mathrm{VS} 13-\mathrm{V} 2$

$G 3=G 2$

$\mathrm{D} 3=\mathrm{D2}$

$\mathrm{T} 3=\mathrm{T} 2$

$P 3=P 2 / 133.3$

WRITE $(6,11) \cup 3, P 3, T 3,63,03, W 3$

WRITE $(6,10)$ ALF

WRITE $(6,12)$ ALF2

$28 \quad D I=D 3$

WRITE $(6,6)$

$99 W 5=W 5-W 5 M$

$W=W 5$

$\mathrm{G} 1=\mathrm{G} 3$

$P 1=P 3 * 133.3$

$T 1=T 3$

$V_{1}=V S 35+U_{3}$

$A I=G 1 * P 1 / D 1$

SHM $=1000.0 * V 1 /(S Q R T(A I))$

$J L=0$

400 CALL PEA2(P1,D1,G1,G2,W,V1,SHM, DR,D2,P2,V2)

$\mathrm{JL}=\mathrm{JL}+1$

CALL TEA2 (P2,D2, G2,T2,ALF, ALF2,ALF3, ALF 4, ALF5)

CALL GEE2 $(T 2, P 2, G Z)$

$G 2=(G Z+3.0 * G 2) / 4.0$

IF (JL.GT.50)G0 TO 500

IF $(A B S(G 2-G Z) . G T .0 .0001)$ GO TO 400

$700 \quad D 5=D 2$ 


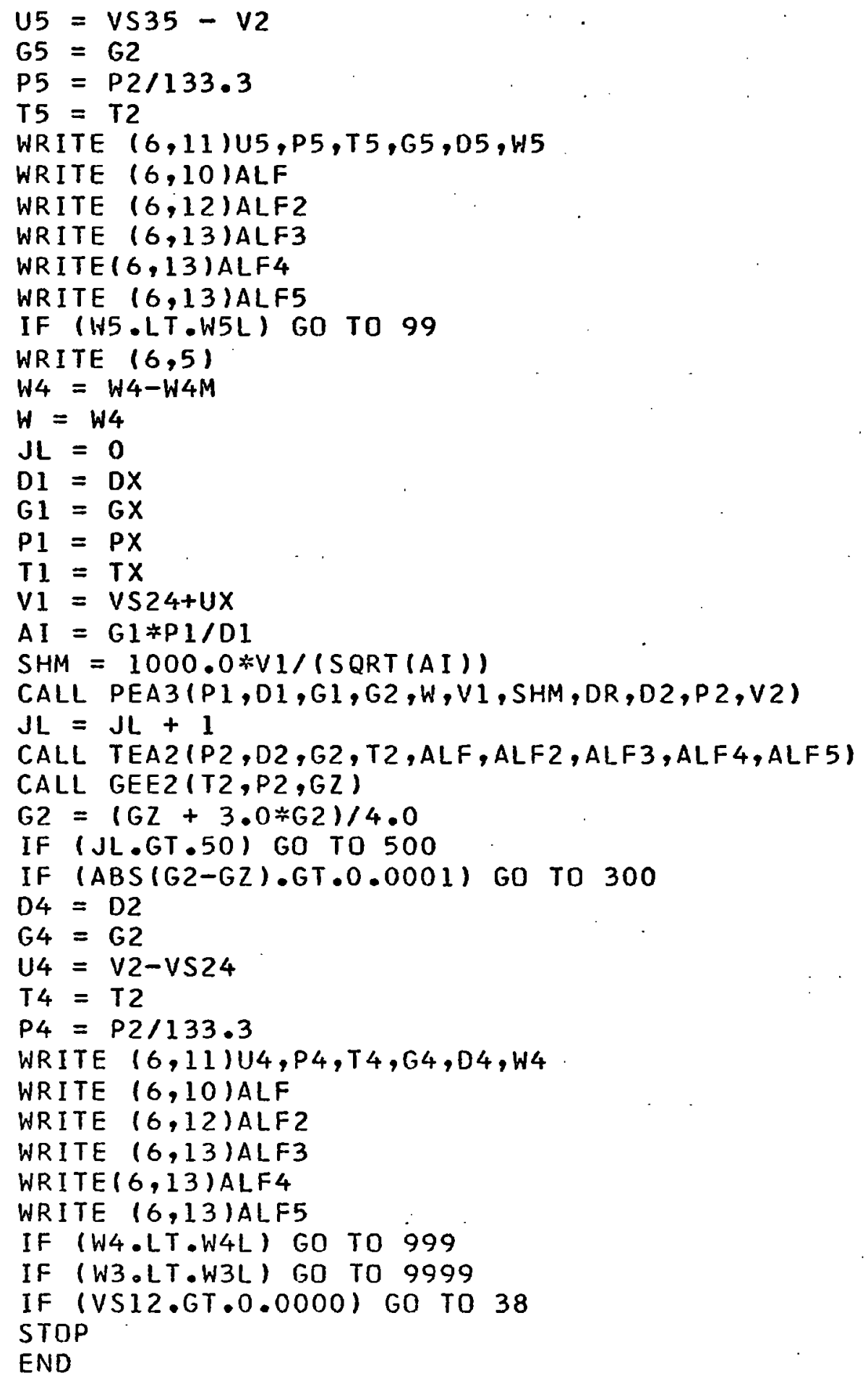


SUBROUT INE TEA2 (P2,D2, GE,T2)

$G 2=G E$
$H=G 2 * P 2 /((G 2-1.0) * D 2)$

REAL MA

$M A=6.63 E-26$

$A L F=(2.3327 E-6) *(H-2.5 * P 2 / 02)$

IF (ALF.GT.100.0) ALF $=100.0$

$A L F 2=(1.3176 E-6) *(H-2.5 * P 2 / D 2)-0.5648 * A L F$

IF (ALF2.GT.100.0) ALF2 $=100.0$

$A L F 3=(8.9758 E-7) *(H-2.5 * P 2 / D 2)-.3725 * A L F-.6812 * A L F 2$

IF (ALF3.GT.100.0) ALF3 $=100.0$

ALF4 $=(5.9961 E-7) *(H-2.5 * P 2 / D 2)-.2488 * A L F-.4551 * A L F 2-.6680 * A L F 3$

IF (ALF4.GT.100.0) ALF $4=100.0$

$B=(4.6891 \mathrm{E}-7) *(\mathrm{H}-2.5 * \mathrm{P} 2 / \mathrm{D2})$

$A L F 5=B-.1946 * A L F-.3559 * A L F 2-.5224 * A L F 3-.7821 * A L F 4$

$A L F A=(A L F+A L F 2+A L F 3+A L F 4+A L F 5) / 100.0$

$T 2=P 2 /(D 2 * 208.1 *(1.0+A L F A))$

RETURN

END 
$-60-$

207

SUBROUT INE PEA3 (P1,D1, G1, G2,W,V $1, S H M, D R, D 2, P 2, V 2)$

$A=G 2 /(S H M * S H M * G 1)-1.0$

$C=W$

$P 2=P 1 *(1.0-G 1 * S H M * S H M * A *(1.0+C) /(G 2+1.0))$

$307 \quad V 2=V 1 *(1.0+A *(1.0+C) /(G 2+1.0))$

$\mathrm{DR}=\mathrm{V} 1 / \mathrm{VZ}$

407

$D 2=D R * D 1$

RETURN

END 
SUBROUTINE PEA2 $(P 1, D 1, G 1, G 2, W, V 1, S H M, D R, D 2, P 2, V 2)$

207

$$
A=G 2 /(S H M * S H M * G 1)-1.0
$$

$B=2.0 *(G 2 * G 2-1.0) * W /(D 1 *(V) * 3))$

$B 1=(G 1-1.0) *((G 2-G) * S H M * S H M) * 2)$

$A 1=2.0 *(G 2+1.0) *(G 1-G 2) * S H M * S H M * G 1$

$E P=A 1 / B 1$

$D=1.0+E P-B /(A * A)$

$C=S Q R T(D)$

$P 2=P 1 *(1.0-G 1 * S H M * S H M * A *(1.0+C) /(G 2+1.0))$

$307 \quad V_{2}=V 1 *(1.0+A *(1.0+C) /(G 2+1.0))$

$D R=V 1 / V 2$

407 RETURN

END 
SUBROUT INE GEE2 $(T 2, P 2, G Z)$

$P 2=P 2 / 133.3$

IF $(T 2 . L T .15000 .0)$ GO TO 15

IF $(T 2 . L T .20000 .0)$ GO TO 25

IF $(T 2 . L T \cdot 21000.0)$ GO TO 35

IF $(T 2 . L T .22000 .0)$ GO TO 45

IF $(T 2 . L T .23000 .0)$ GO TO 55

IF $(T 2 . L T .24000 .0)$ GO TO 65

IF $(T 2 . L T .25000 .0)$ GO TO 75

IF (T2.LT.26000.0) GO TO 85

IF $(T 2 . L T .27000 .0)$ GO TO 95

IF $(T 2 . L T .28000 .0)$ GO TO 105

IF (T2.LT.29000 0) GO TO 115

IF $(T 2 . L T .30000 .0)$ GO TO 125

$G A=1.125$

$G B=1.130$

$G C=1.140$

$G D=1.160$

$G E=1.193$

GO TO 1000

25 IF $(T 2 . L T .16000 .0)$ GO TO 135

IF $(T 2 . L T .17000 .0)$ GO TO 145

IF $(T 2 . L T .18000 .0)$ GO TO 155

IF (T2.LT.19000.0) GO TO 165

$G A=1.118-0.175 * A L O G(T 2 / 20000.0)$

$G B=1.148-0.039 * A L O G(T 2 / 20000.0)$

$G C=1.162+0.078 * A L O G(T 2 / 20000.0)$

$G D=1.170-0.039 * A L O G(T 2 / 20000.0)$

$G E=1.207-0.234 * A L O G(T 2 / 20000.0)$

GO TO 1000

15 IF (T2.LT.9000.0) GO.TO 175

IF $(T 2 . L T .10000 .0)$ GO TO 185

IF $(T 2 . L T .11000 .0)$ GO TO 195

IF (T2.LT.12000.0) GO TO 205

IF (T2.LT.13000.0) GO TO 215

IF $(T 2 . L T .14000 .0)$ GO TO 225

$G A=1.131+0.072 * A L O G(T 2 / 15000.0)$

$G B=1.135$

$G C=1.157-0.420 * A L O G(T 2 / 15000.0)$

$G D=1.257-0.826 * A L O G(T 2 / 15000.0)$

$G E=1.367-1.000 * A \operatorname{LOG}(T 2 / 15000.0)$

GO TO 1000

175 IF (T2.LT.3000.0) GO TO 235

IF $(T 2 . L T .4000 .0)$ GO TO 245

IF $(T 2 . L T .5000 .0)$ GO TO 255

IF $(T 2 . L T .6000 .0)$ GO TO 265

IF $(T 2 . L T .7000 .0)$ GO TO 275

IF $(T 2 . L T .8000 .0)$ GO TO 285 


$$
\begin{aligned}
& G A=1.363-1.452 * A L O G(T 2 / 9000.0) \\
& G B=1.546-0.637 * A L O G(T 2 / 9000.0) \\
& G C=1.609-0.375 * A L O G(T 2 / 9000.0) \\
& G D=1.660-0.051 * A L O G(T 2 / 9000.0) \\
& G E=1.665-0.008 * A L O G(T 2 / 9000.0) \\
& \text { GO TO } 1000 \\
& 125 \quad G A=1.125 \\
& G B=1.130 \\
& G C=1.140 \\
& G D=1.160-0.118 * A L O G(T 2 / 30000.0) \\
& G E=1.193-0.059 * A L O G(T 2 / 30000.0) \\
& \text { GO TO } 1000 \\
& 115 \quad G A=1.125 \\
& \mathrm{~GB}=1.130 \\
& G C=1.140 \\
& G D=1.164-0.142 * A L O G(T 2 / 29000.0) \\
& G E=1.195-0.057 * A L O G(T 2 / 29000.0) \\
& \text { GO TO } 1000 \\
& 105 \quad G A=1.125 \\
& G B=1.130+0.055 * A L O G(T 2 / 28000.0) \\
& G C=1.140 \\
& G D=1.169-0.165 * A L O G(T 2 / 28000.0) \\
& G E=1.197-0.055 * A L O G(T 2 / 28000.0) \\
& \text { GO TO } 1000 \\
& 95 \quad \mathrm{GA}=1.125 \\
& G B=1.128+0.053 * A \operatorname{LOG}(T 2 / 27000.0) \\
& G C=1.140-0.106 * A L O G(T 2 / 27000.0) \\
& G D=1.175-0.159 \% A L O G(T 2 / 27000.0) \\
& G E=1.199-0.026 * A L O G(T 2 / 27000.0) \\
& \text { GO TO } 1000 \\
& 85 \quad G A=1.125 \\
& G B=1.126+0.051 * A \operatorname{LOG}(T 2 / 26000.0) \\
& G C=1.144-0.127 * A L O G(T 2 / 26000.0) \\
& G D=1.181-0.051 * A L O G(T 2 / 26000.0) \\
& G E=1.200 \\
& \text { GO TO } 1000 \\
& 75 \quad \mathrm{GA}=1.125+0.049 * A \operatorname{LOG}(\mathrm{T} 2 / 25000.0) \\
& G B=1.124+0.024 * A \operatorname{LOG}(T 2 / 25000.0) \\
& G C=1.149-0.171 * A L D G(T 2 / 25000.0) \\
& G D=1.183 \\
& G E=1.2000+0.122 * A L O G(T 2 / 25000.0) \\
& \text { GO TO } 1000 \\
& 65 \quad G A=1.123+0.094 * A L D G(T 2 / 24000.0) \\
& G B=1.123-0.094 * A L O G(T 2 / 24000.0) \\
& G C=1.156-0.164 * A L O G(T 2 / 24000.0) \\
& G D=1.183 \\
& G E=1.195+0.047 \% A L O G(T 2 / 24000.0) \\
& \text { GO TO } 1000
\end{aligned}
$$


$G A=1.119+0.045 * A L O G(T 2 / 23000.0)$

$G B=1.127-0.157 * A L O G(T 2 / 23000.0)$

$G C=1.163-0.067 * A L O G(T 2 / 23000.0)$

$G D=1.183+0.067 * A L O G(T 2 / 23000.0)$

$G E=1.193-0.022 * A \operatorname{LOG}(T 2 / 23000.0)$

GO TO 1000

$G A=1.117+0.021 * A L D G(T 2 / 22000.0)$

$G B=1.134-0.193 * A \operatorname{LOG}(T 2 / 22000.0)$

$G C=1.166+0.043 * A L O G(T 2 / 22000.0)$

$G D=1.180+0.086 * A L O G(T 2 / 22000.0)$

$G E=1.194-0.107 * A L O G(T 2 / 22000.0)$

GO TO 1000

$G A=1.116-0.041 * A L O G(T 2 / 21000.0)$

$G B=1.143-0.102 * A L O G(T 2 / 21000.0)$

$G C=1.164+0.041 * A L O G(T 2 / 21000.0)$

$G D=1.176-0.123 * A L O G(T 2 / 21000.0)$

$G E=1.199-0.164 * A L O G(T 2 / 21000.0)$

GO TO 1000

165

$G A=1.127-0.148 * A \operatorname{LOG}(T 2 / 19000.0)$

$\mathrm{GB}=1.150$

$G C=1.158+0.037 * A L O G(T 2 / 19000.0)$

$G D=1.172-0.111 * A \operatorname{LOG}(T 2 / 19000.0)$

$G E=1.219-0.444 * A L O G(T 2 / 19000.0)$

GO TO 1000

$155 \mathrm{GA}=1.135-0.070 * A \operatorname{LOG}(T 2 / 18000.0)$

$G B=1.150+0.052 * A \operatorname{LOG}(T 2 / 18000.0)$

$G C=1.156+0.122 * A L O G(T 2 / 18000.0)$

$G D=1.178-0.280 * A L O G(T 2 / 18000.0)$

$G E=1.243-0.630 * A L O G(T 2 / 18000.0)$

GO TO 1000

$145 \quad G A=1.139+0.049 * A L O G(T 2 / 17000.0)$

$G B=1.147+0.115 * A L O G(T 2 / 17000.0)$

$G C=1.149+0.033 * A L O G(T 2 / 17000.0)$

$G D=1.194-0.363 * A \operatorname{LOG}(T 2 / 17000.0)$

$G E=1.279-0.660 * A L O G(T 2 / 17000.0)$

GO TO 1000

$135 G A=1.136+0.077 * A L O G(T 2 / 16000.0)$

$G B=1.140+0.077 * A L O G(T 2 / 16000.0)$

$G C=1.147-0.155 * A L O G(T 2 / 16000.0)$

$G D=1.216-0.635 * A L O G(T 2 / 16000.0)$

$G E=1.319-0.743 \% A L O G(T 2 / 16000.0)$

GO TO 1000

$225 \quad G A=1.126+0.054 * A L O G(T 2 / 14000.0)$

$G B=1.135-0.189 * A L O G(T 2 / 14000.0)$

$G C=1.186-0.661 * A L O G(T 2 / 14000.0)$

$G D=1.314-1.093 * A L O G(T 2 / 14000.0)$

$\mathrm{GE}=1.436-0.877 * A \operatorname{LOG}\left(\mathrm{T}_{2} / 14000.0\right)$

GO TO 1000 
$G A=1.122-0.012 * A \operatorname{LOG}(T 2 / 13000: 0)$

$G B=1.149-0.412 * A L D G(T 2 / 13000.0)$

$G C=1.235-1.012 * A L O G(T 2 / 13000.0)$

$G D=1.395-1.124 * A L O G(T 2 / 13000.0)$

$G E=1.501-0.837 * A L O G(T 2 / 13000.0)$

205

GO TO 1000

$G A=1.123-0.310 * A \operatorname{LOG}(T 2 / 12000.0)$

$G B=1.183-0.839 * A L O G(T 2 / 12000.0)$

$G C=1.316-1.253 * A L O G(T 2 / 12000.0)$

$G D=1.485-0.942 * A L O G(T 2 / 12000.0)$

$G E=1.568-0.517 * A \operatorname{LOG}(T 2 / 12000.0)$

$195 \mathrm{GA}=1.150-0.703 * A L O G(T 2 / 11000.0)$

$G B=1.255-1.416 * A L O G(T 2 / 11000.0)$

$G C=1.425-1.228 * A L O G(T 2 / 11000.0)$

$G D=1.567-0.283 * A L O G(T 2 / 11000.0)$

$G E=1.613-0.346 * A L O G(T 2 / 11000.0)$

GO TO 1000

$185 \quad G A=1.217-1.386 * A \operatorname{LOG}(T 2 / 10000.0)$

$G B=1.390-1.481 * A L O G(T 2 / 10000.0)$

$G C=1.542-0.636 * A L O G(T 2 / 10000.0)$

$G D=1.640-0.190 * A L O G(T 2 / 10000.0)$

$G E=1.646-0.085 * A L O G(T 2 / 10000.0)$

GO TO 1000

$285 \quad G A=1.537-0.524 * A L O G(T 2 / 8000.0)$

$G B=1.621-0.195 * A L O G(T 2 / 8000.0)$

$G C=1.651-0.075 * A L O G(T 2 / 8000.0)$

$G D=1.666$

$G E=1.666$

GO TO 1000

$275 \quad G A=1.607-0.175 * A L D G(T 2 / 7000.0)$

$G B=1.647-0.078 * A L O G(T 2 / 7000.0)$

$G C=1.661-0.032 \div A \operatorname{LOG}(T 2 / 7000.0)$

$G D=1.666$

$\mathrm{GE}=1.666$

GO TO 1000

$265 \quad G A=1.634-0.093 * A L O G(T 2 / 6000.0)$

$G B=1.659-0.038 * A L O G(T 2 / 6000.0)$

$G C=1.666$

$G D=1.666$

$G E=1.666$

GO TO 1000

$255 \quad G A=1.651-0.036 * A L O G(T 2 / 5000.0)$

$G B=1.666$

$G C=1.666$

$\mathrm{GD}=1.666$

$G E=1.666$

GO TO 1000 


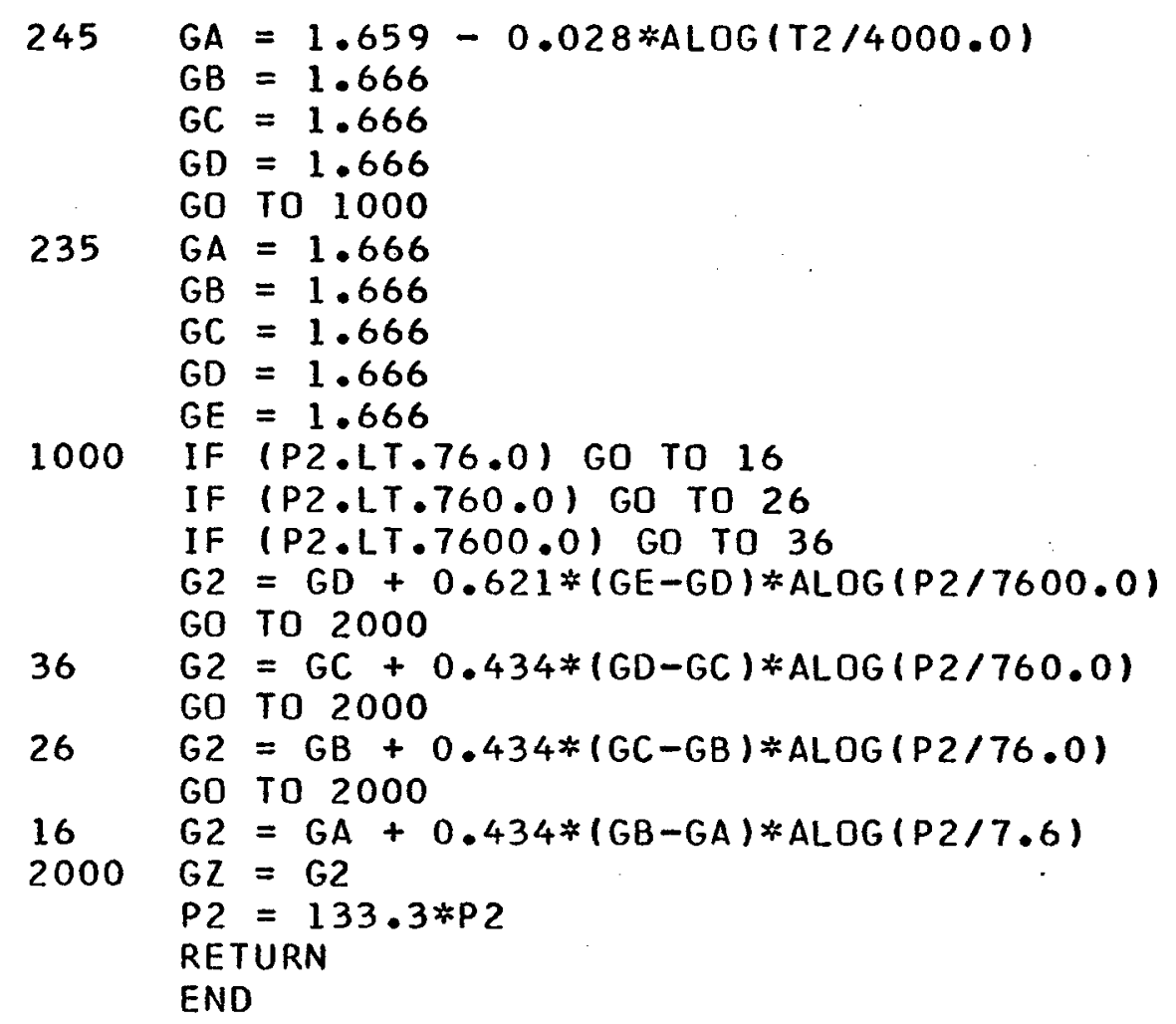


$-67-$

Appendix C

CONTINUOUSLY VARYING PARAMETERS

The numerical method to describe continuously varying parameters is extremely simple. Essentially, the set of equations,

$$
\begin{aligned}
& \Delta V_{\alpha}=\Delta \mu_{1}+f_{\alpha} \Delta \rho_{1}+h_{\alpha} \Delta p_{1} \\
& \Delta V_{\beta}=\Delta \mu_{1}+f_{\beta} \Delta \rho_{1}+h_{\beta} \Delta p_{1} \\
& \Delta V_{\gamma}=\Delta \mu_{1}+f_{\gamma} \Delta \rho_{1}+h_{\gamma} \Delta p_{1}
\end{aligned}
$$

are inverted to obtain

$$
\begin{aligned}
& p_{1}(x)=p_{1}(0)+\frac{\Delta v_{\gamma}-\Delta v_{\alpha}-\frac{f_{r}-f_{\alpha}}{f_{s}-f_{\alpha}}\left(\Delta v_{s}-\Delta v_{\alpha}\right)}{h_{\gamma}-h_{\alpha}-\frac{f_{\gamma}-f_{\alpha}}{f_{s}-f_{\alpha}}\left(h_{\beta}-h_{\alpha}\right)} \\
& \rho_{1}(x)=\rho_{1}(0)+\frac{\Delta v_{\beta}-\Delta v_{\alpha}-\left(h_{\beta}-h_{\alpha}\right) \Delta p_{1}}{f_{\beta}-f_{\alpha}} \\
& \mu_{1}(x)=\mu_{1}(0)+\Delta v_{\alpha}-f_{\alpha} \Delta \rho_{1}-h_{\alpha} \Delta p_{1}
\end{aligned}
$$

Using a simple predictor integration formula for $f$ and $h$ proved to be too unstable a method. It is unsuccessful because equations (C-2) involve the differences of differences, and because the flow field produced by the light source is not as reproducible as we would like. Instead of finding an exact solution, simple limits were found on the variation of the 
$-68-$

parameters. The lower limit was found by assuming that one parameter alone was responsible for the variation in shock velocity. For example,

$$
\Delta p_{1}=\Delta v_{\gamma} \cdot h_{\gamma}
$$

while the upper limit for $p_{1}$ would then be

$$
\begin{aligned}
& \Delta \mu_{1}=\Delta v_{\alpha} \\
& \Delta \rho_{1}=\left(\Delta v_{\beta}-\Delta \mu_{1}\right) / f_{\beta} \\
& \Delta p_{1}=\Delta v_{\gamma} / h_{\gamma}-\left(\Delta \mu_{1}+f_{\gamma} \Delta \rho_{1}\right) / a_{\gamma}
\end{aligned}
$$

since the other two parameters would account for the complete shock variation on the other two measurements.

In our particular case, the discontinuity $\langle 3,5\rangle$ is not a shock wave because there is energy input into state 5 . In fact, $W_{s}$ would be very close to the Chapman - Jouguet energy. Therefore, we re-derive the partial derivatives $\frac{\partial V}{\partial R_{1}}$ and $\frac{\partial V}{\partial \rho_{1}}$, assuming that the front $\langle 3,5\rangle$ is at the Chapman - Jouguet point.

$$
V=\mu_{1}+\sqrt{\frac{p_{1}}{s_{1}}\left[(z-1)\left(g_{2}+1\right)-g_{2}\right]}
$$


$-69-$

$$
\frac{\partial V}{\partial p_{1}}=\frac{V-\mu_{1}}{\partial p_{1}}+\frac{\left(g_{2}+1\right) p_{1}}{\left(v-\mu_{1}\right) \rho_{1}} \frac{d z}{d p_{1}}
$$

$$
\frac{\partial v}{\partial \rho_{1}}=-\frac{\left(v-\mu_{1}\right)}{\partial \rho_{1}}+\frac{\left(g_{2}+1\right) p_{1}}{\left(v-\mu_{1}\right) \rho_{1}} \frac{d z}{d \rho_{1}}
$$

At the Chapman - Jouguet point,

$$
z_{\text {C.J. }}=\frac{1}{2} z_{\text {SHOCK }}
$$

Therefore,

$$
\frac{d z_{C . J_{1}}}{d p_{1}}=\frac{1}{2} \frac{\alpha z_{\text {sHock }}}{d p_{1}}
$$

and

$$
\frac{d z_{C . J}}{d \rho_{1}}=\frac{1}{2} \frac{d z_{\text {SHOCK }}}{d \rho_{1}}
$$

where $\frac{d z}{d p_{1}}$ SHock and $\frac{d z_{\text {sHock }}}{d s_{1}}$ as derived in chapter three.

Figure $\mathrm{C}-1$ indicates the variation of four shock velocities throughout the flow field. These velocities have been normalied for the distance which they travel into the flow field. 


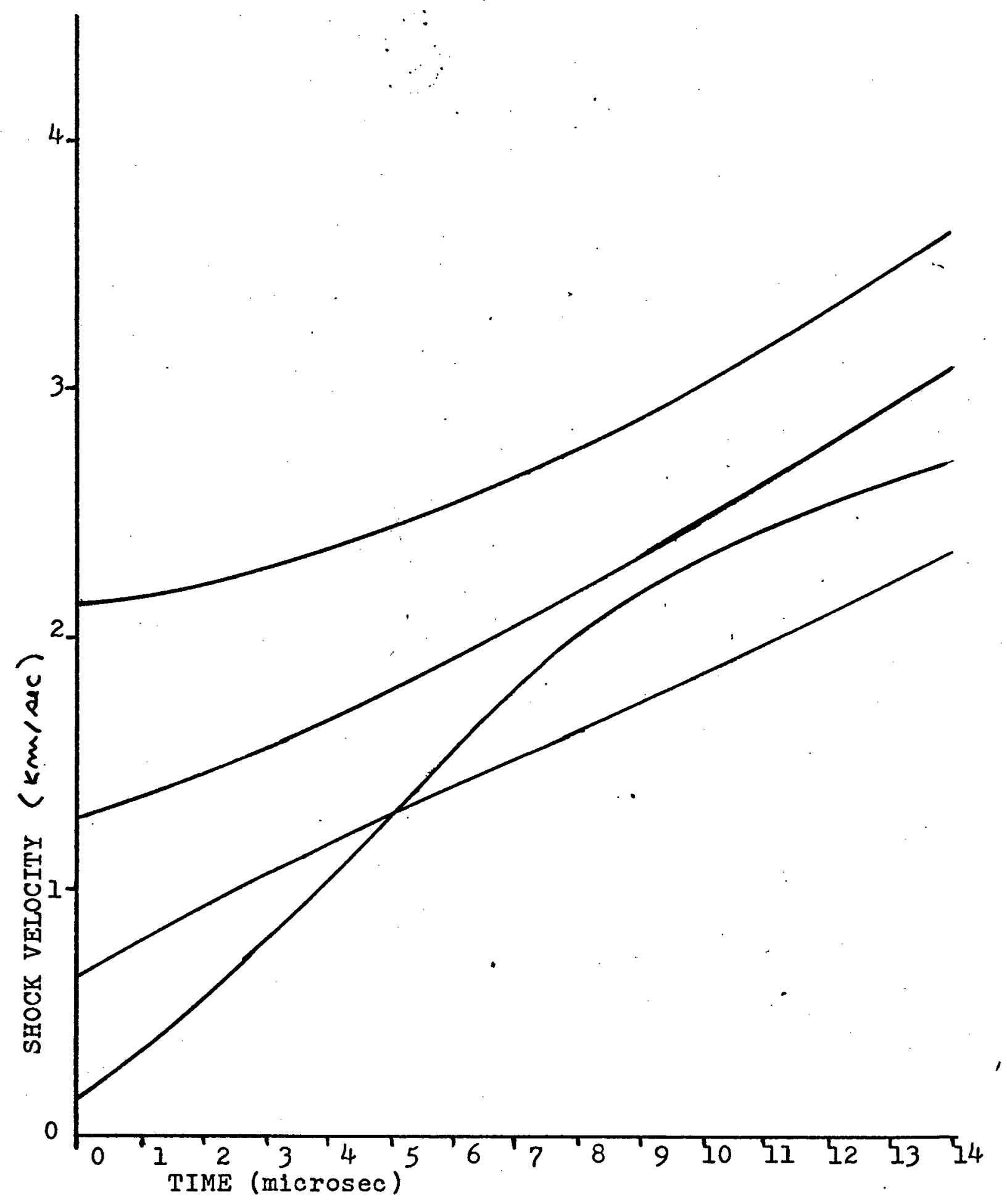

Fig. C Shock Velocity Behind Radiation Front 
1 FORMAT $(5 X, I 2,5 X, F 6: 3,5 X, F 6,1,5 X, F 8,6,5 X, F 8,1,5 X, F 6,3)$

FORMAT $(5 X, 1 H I, 6 X, 2 H U 3,9 X, 2 H P 3,9 X, 2 H D 3,11 X, 2 H T 3,9 X, 2 H G 3)$

FORMAT ( $12,4 \mathrm{~F} 6.3)$

FORMAT $(5 X, 12,5 X, F 6.3,5 X, F 6.3,5 X, F 6.3)$

FORMAT (I 2 )

FORMAT (F6.3)

$\operatorname{READ}(5,7) \mathrm{GE}$

$I M=17$

DIMENSION $V 1(17), V_{2}(17), V_{3}(17), V \times(17), V 4(17)$

DIMENSION U1 (17),P1(17),D1 (17),T $1(17), G 2(17)$

$\operatorname{READ}(5,1) I, U 1(1), P 1(1), D 1(1), T_{1}(1), G 1$

$G 2(I)=G 1$

WRITE $(6,2)$

WRITE $(6,1) I, U 1(I), P I(I), D 1(I), T 1(I), G 1$

$P 1(I)=133.3 \% P 1(1)$

$\operatorname{READ}(5,3) I, V 1(I), V 2(I), V 3(I), V 4(I)$

100

$\operatorname{READ}(5,3) I, V 1(I), V 2(I), V 3(I), V 4(I)$

$G 2(I)=G 1$

$V X(I)=V 1(I)$

CALL FEE $(G 2(I-1), V X(I), U 1(I-1), D 1(I-1), P I(I-1), F X)$

CALL GEE $(G 2(I-1), V X(I), U 1(I-1), D 1(I-1), P I(I-1), G X)$

$F G 1=G X$

$F 1=F X$

$V X(I)=V 2(I)$

CALL FEE (G2 $(I-1), V X(I), U 1(I-1), D 1(I-1), P 1(I-1), F X)$

CALL GEE $(G 2(I-1), V X(I), U 1(I-1), D 1(1-1), P 1(I-1), G X)$

$F 2=F X$

$F G 2=G X$

$V X(I)=V 3(I)$

CALL FEE $(G 2(I-1), V X(I), U 1(I-1), D 1(1-1), P I(I-1), F X)$

CALL GEE $(G 2(I-1), V X(I), U 1(I-1), D 1(I-1), P 1(I-1), G X)$

$F 3=F X$

$F G 3=G X$

$V X(I)=V 4(I)$

CALL FEE $(G 2(I-1), V X(I), U 1(I-1), D 1(I-1), P 1(I-1), F X)$

CALL GEE $(G 2(I-1), V X(I), U 1(I-1), D 1(I-1), P 1(I-1), G X)$

$F G 4=G X$

$F 4=F X$

$U 1(I)=U 1(I-1)+V 1(I)-V 1(I-1)$

$D 1(I)=D 1(I-1)+(V 4(I)-V 4(I-1)) / F 4-(U 1(I)-U 1(I-1)) / F 4$

$X X=(U 1(I)-U 1(I-1)) / F G 2+(D 1(I)-D 1(I-1)) / F G 2$

$P 1(I)=P 1(I-1)-(V 2(I)-V 2(I-1)) / F G 2+X X$

$P 2=P 1(I)$

$\mathrm{D} 2=\mathrm{D} 1(\mathrm{I})$

CALL TEA2 $(P 2, D 2, G E, T 2)$

$T 1(I)=T 2$

$P 1(I)=P 1(I) / 133.3$

WRITE $(6,1) I, U 1(I), P 1(I), D 1(I), T I(I), G I$

$P 1(I)=133.3 * P 1(I)$

IF (I.LT.IM) G0 TO 100

STOP

END 
SUBROUT INE GEE $(G 2, V X, U 1, D 1, P 1, G X)$

$$
\begin{aligned}
& \text { LAM }=(G 2-1.0) /(G Z+1.0) \\
& Z=(1.0-L A M) *((V X-U 1) * 2) * D 1 / P 1-L A M \\
& C A=S Q R T(Z *(1.0+L A M * Z) /(1.0+L A M)) \\
& C D=(Z-1.0) /(2.0 *(L A M+Z)) \\
& C C=-C A / Z-1.0 \\
& C B=(Z-1.0) / 2.0 \\
& D Z=(C A+C B) /(P 1 *(C C+C D)) \\
& D Z=0.5 * D Z \\
& G X=(V X-U 1) *(0.5 / P 1+0.5 * D Z /(Z-1.0))
\end{aligned}
$$

RETURN

END 
SUBROUT INE FEE $(G 2, V X, U 1,01, P 1, F X)$

$L A M=(G 2-1.0) /(G 2+1.0)$

$Z=(1.0-L A M) *((V X-U 1) * 2) * 01 / P 1-L A M$

$C A=-S Q R T((1.0+L A M * Z) /(Z *(1.0+L A M)))$

$C B=1.0-(Z-1.0) /(2.0 *(L A M+Z))$

$D Z=(Z-1.0) /(2.0 * D 1 *(C A+C B))$

$D Z=0.5 * D Z$

$F X=(V X-U 1) *(-.5 / 01+0.5 * D Z /(Z-1.0))$

RETURN

END 
Appendix D

THE SHOCK TUBE

In this work, the original "low attenuation" shock tube of P. R. Smy $(1962,1965)$ was used. The driver gas is electrically heated and accelerated by a standard back-strap configuration, and a mylar membrane is employed to separate test and driver gases. This shock tube has been used in several experiments (ref. 4, 11, 12, 13, 25, 30). Details of design and performance are given in Smy's article (1965) and also in M. Phillips' thesis (1969). In order to use this shock tube for the testing of some other gas flow, one needs to ascertain that a shock is actually formed. Smy's explanation was not entirely satisfactory and when coupled with the criticisms of the conventional electromagnetic shock tubes by Muntenbruch (1969), and the discussion of flow instabilities in electric arc-driven tubes by Barach and Vermillion (1965), serious doubt arose about the description of the shock tube and the predictability of any shock waves. The recent work of Phillips has helped to understand the performance of such an arc-driven membrane shock tube. However, since Phillips introduced several modifications in the design of the tube, which were not incorporated in our tube, we have establised. independently, that in our tube, a reproducible and well behaved shock is formed.

In order to intensify our understanding of the performance of the arc-driven shock tube, we first derive the shock velocity 
as a function of the energy stored in the driving capacitor bank. The total kinetic energy, $\frac{1}{2} M_{m} \mu_{0}^{2}$, introduced through the membrane into the test section of the shock tube must be a fraction $\beta$ of the capacitor bank energy, $\frac{1}{2} \subset V_{c}^{2}$,

$$
\frac{1}{2} M_{m} \mu_{0}^{2}=\beta \frac{1}{2} C V_{c}^{2}
$$

$M$ is the mass originally expelled by the discharge. $\mu_{0}$ is the average particle velocity at the membrane, but since more and more mass is accelerated and the total momentum is constant, the particle velocity will gradually decrease;

$$
\mu(x)\left(M_{m}+A x S_{1}\right)=\mu_{0} M_{m}
$$

where $A$ is the cross-sectional area, and $x$ is the distance from the membrane. Thus, one obtains the contact-surface velocity as a function of position

$$
\mu(x)=\beta V_{c} \sqrt{\frac{c}{M_{m}}} \frac{1}{1+\frac{A \times S_{1}}{M_{m}}}
$$

Smy derived an identical expression, treating the driver gas as a bullet and considering only integrated magnetic driving effects.

With this background and the motivation of understanding the properties of the shock tube, we have two objectives: firstly, to establish that a shock front is formed and that its properties correspond to those given by solution of the 
Rankine - Hugoniot equations, and secondly, to test the functional dependence suggested in equation (D-3).

In order to test the quality of the shock waves formed, we measure the contact surface and shock velocities (Phillips, 1969). Ideally, the contact surface velocity should equal the particle velocity in the shock heated gas. In practice, boundary layers at the wall of the shock tube result in a mass flow out of the region between the contact surface and shock front. Thus, the particles have a net velocity towards the contact surface so that the particle velocity is somewhat smaller than the contact surface velocity. In typical smear pictures, the contact surface and shock front can be easily ldentified (fig. 9). Therefore, both velocities are measureable. In figures D-I and D-2 the contact surface velocity is plotted against the shock velocity. The contact surface velocity has been obtained in three ways:

1. by measuring the contact surface velocity,

2. by measuring the shock velocity; calculating the particle velocity from the Rankine - Hugoniot equations and identifying this with the contact surface velocity, as for the ideal case, and

3. by measuring the shock velocity and applying the well-known boundary layer theory of Roshko (1960) to calculate the contact surface velocity.

The agreement is quite good, so that on the basis of these measurements, the flow pattern looks like a shock wave with 

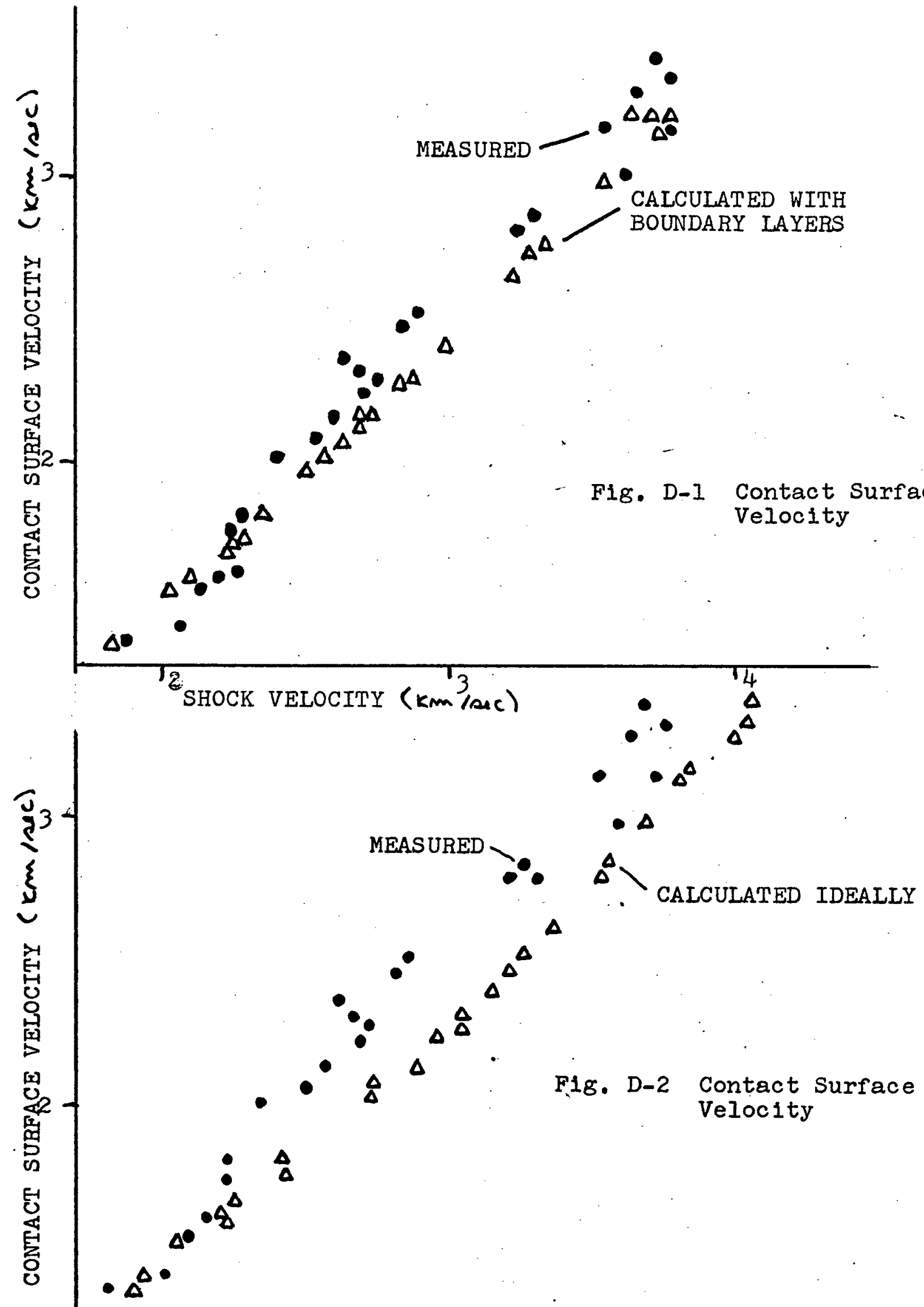

SHOCK VELOCITY $\left(\mathrm{km}^{1 / 3} / \mathrm{sec}\right)$ 
boundary layers in the investigated initial pressure range of 1 to 10 Torr. A further indication of the formation of $a$ shock front is provided by a vertical smear picture (fig. D-3) which clearly shows a planar shock front followed by a diffuse contact surface.

We now proceed to test the attenuation equation (D-3). There are two convenient ways to test this relation, since the contact surface velocity depends upon the two external parameters, $V_{C}$ and $\rho_{1}$. Figure $D-4$ demonstrates the variation of the contact surface velocity with the bank voltage $V_{c}$ and indicates primarily that there is a large variation in the shock velocity formed under identical conditions. The expected proportion is also roughly indicated.

Figures $D-5, D-6$, and $D-7$ show the variation of the contact surface velocity with the downstream pressure. The striking result is that $M_{m}$ cannot be the total mass of driver gas as Smy has predicted. In trying to acquire a better fit, it was noticed that no single value of $M_{m}$ would cause equation D-3 to correspond to the experimental curves. However, at lower downstream pressures, a good fit could be obtained by taking $M_{m}=M_{1} \sim$ the mass of driver gas originally between the two electrodes. At higher pressures, a better fit was obtained by taking $M_{M}$ to be larger; $M_{M}=M_{2} \sim$ one-half of the original total driver gas. A seemingly reasonable explanation for this behaviour would be that capacitor ringing causes a perturbation in the driving mechanism. These ringing effects are a common 

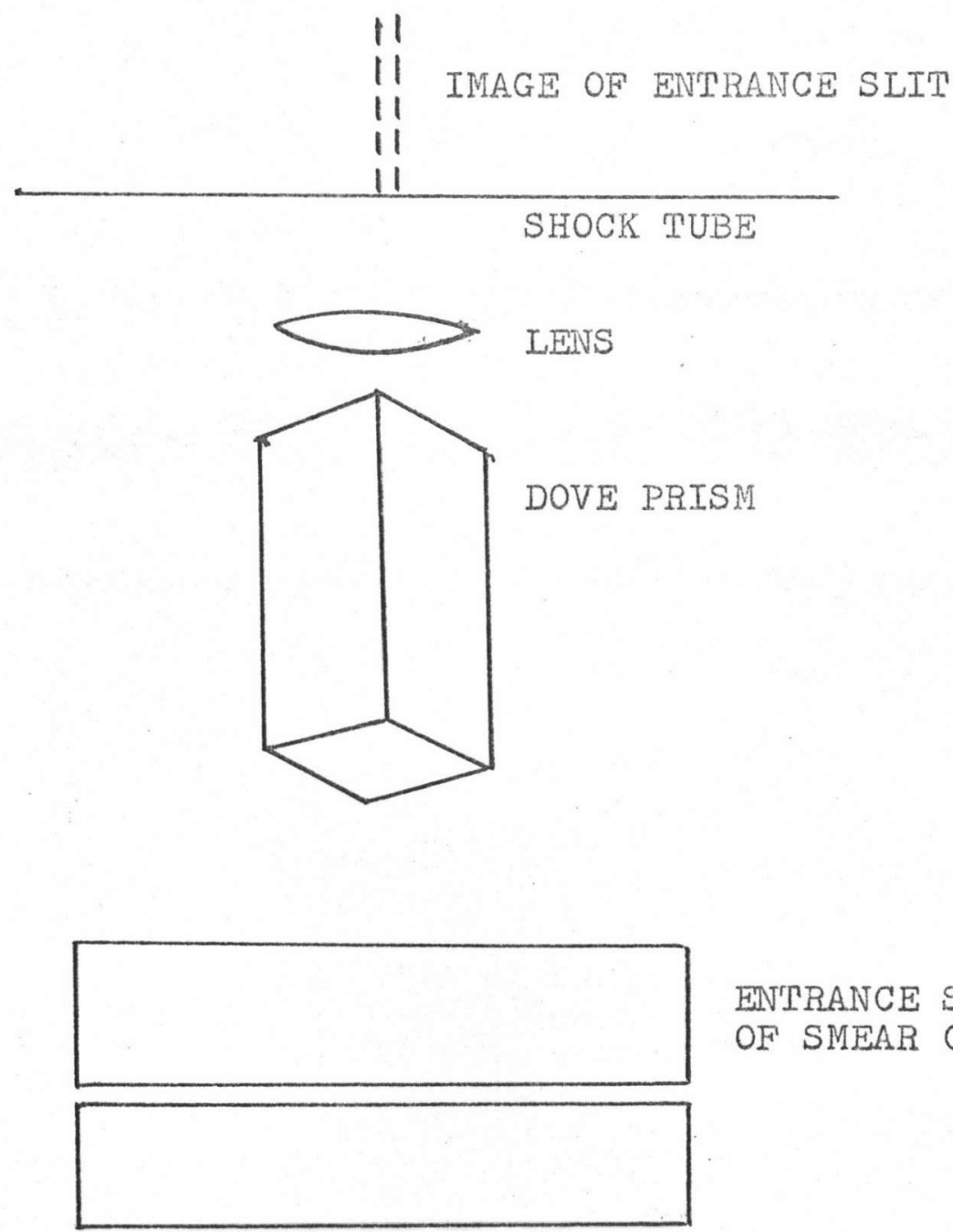

ENTRANCE SIIT

OF SMEAR CAMERA
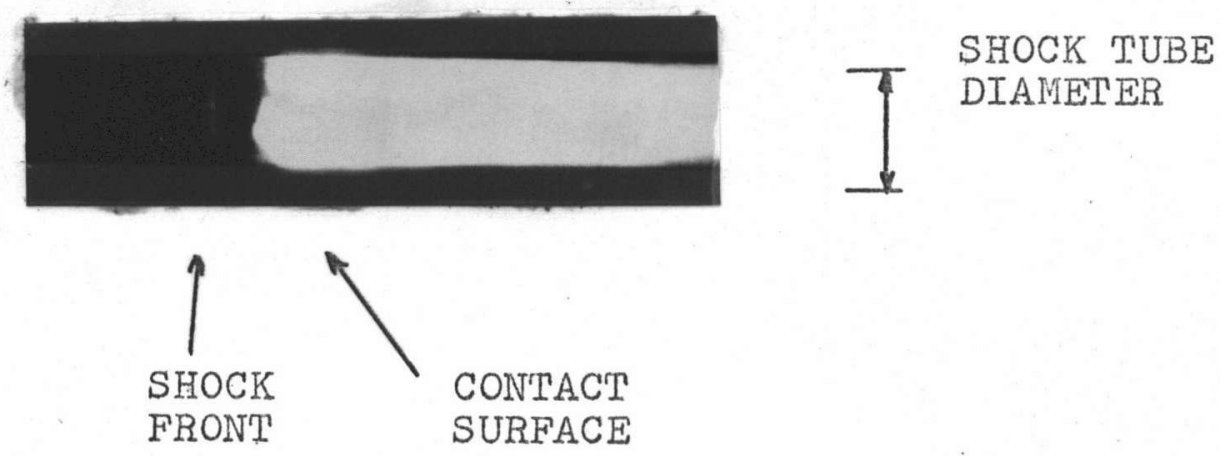

Fig. D 3 Vertical Smear Picture 
$-80-$



Fig. D-4 Shock Velocity vs $V_{c}$ 
F18. D-5 Shock Attenuation

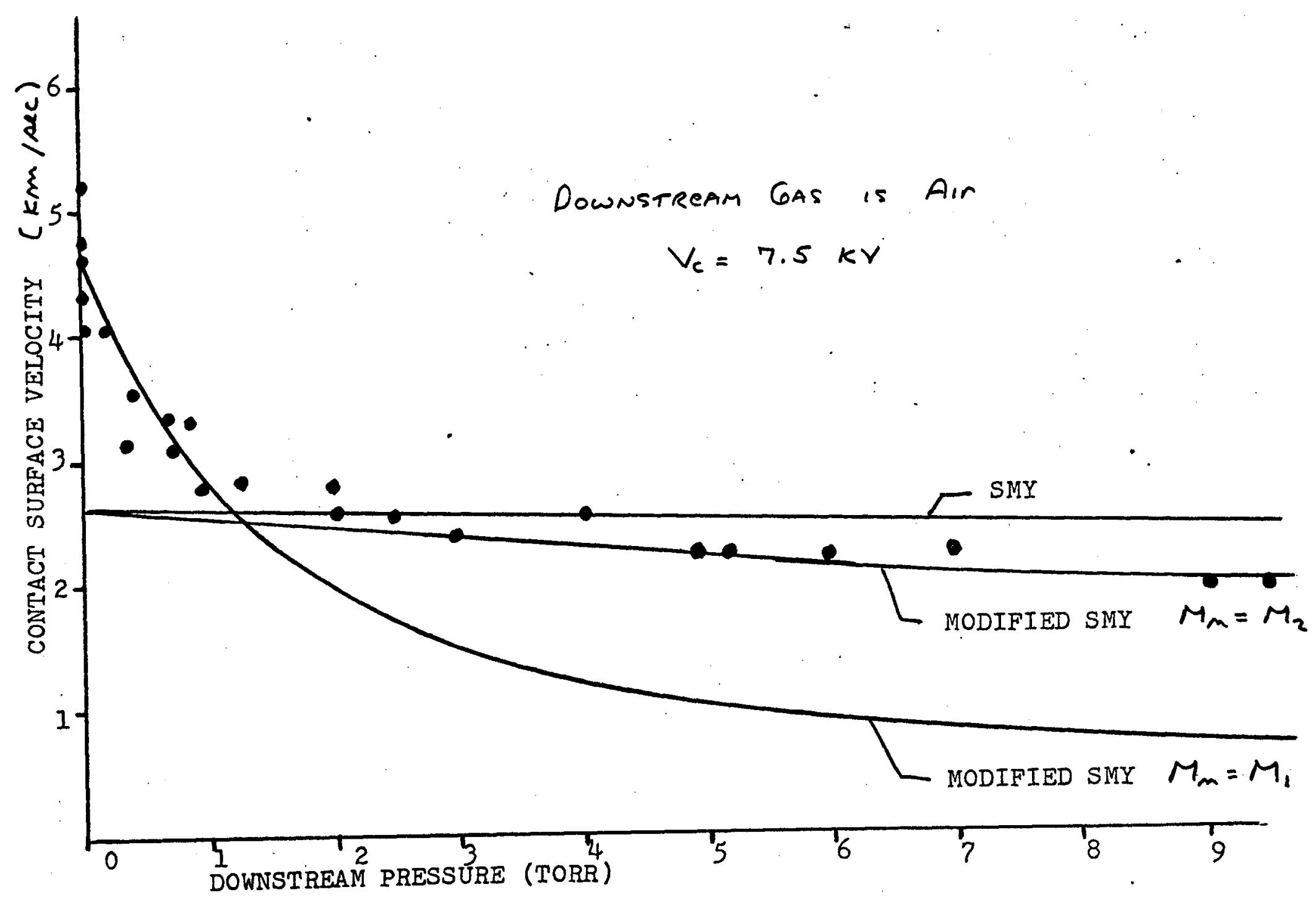




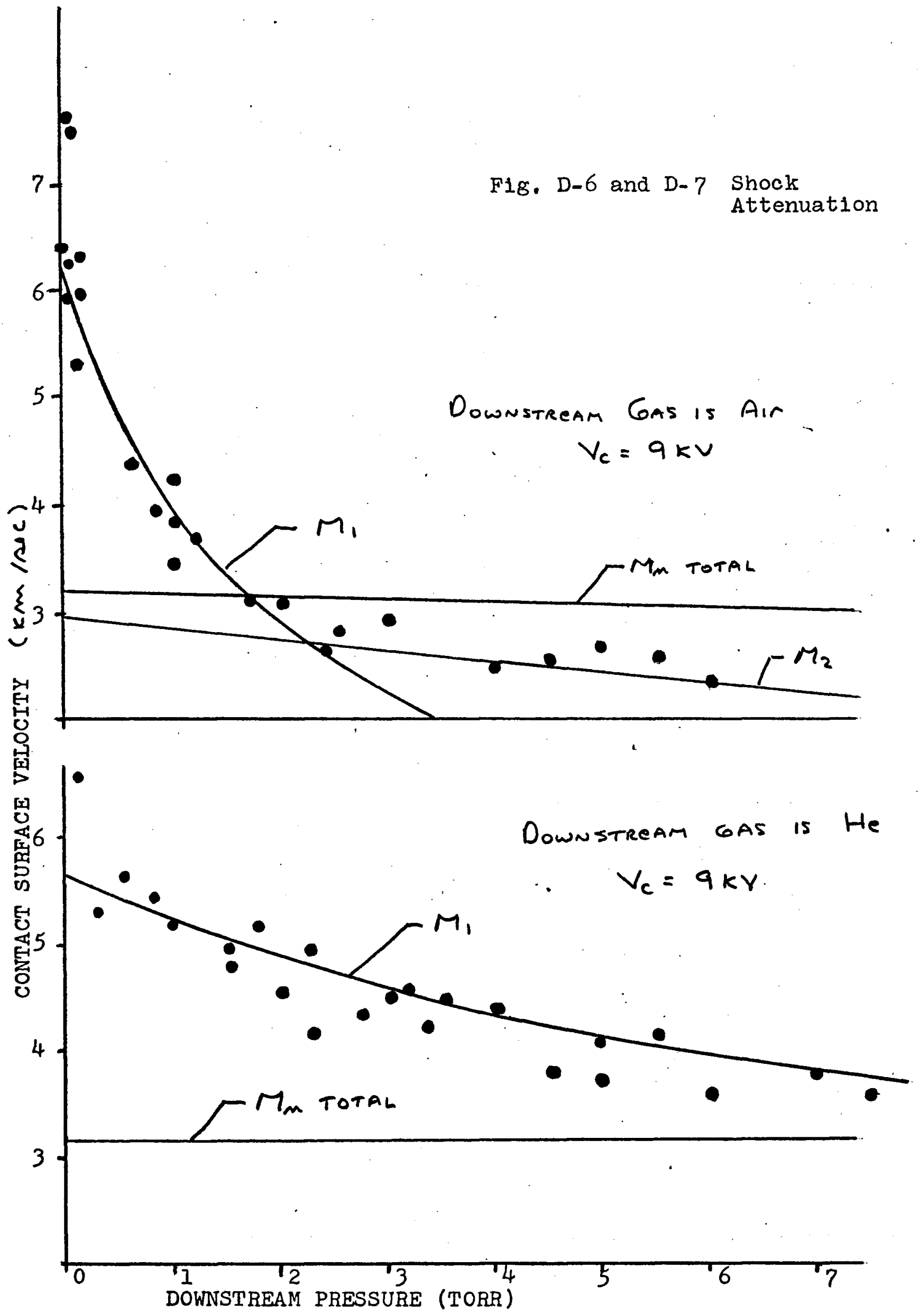


problem in electromagnetic shock tubes (Muntenbruch, 1969). In order to test this hypothesis, we applied a crowbar to the discharge (fig. D-8). Although the crowbar was not completely successful, one of the smear pictures yielded just the desired information. As can be clearly seen, the partial crowbar reduces the secondary ringing effects enough so that the takeover of the driving mechanism is distinct. The partial crowbar results compare quite well with the modified theory (fig. D-9), and establish not only that the flow field is perturbed by capacitor ringing, but also that equation D-3 holds.

In conclusion, the shock tube provides a low attenuation, high velocity shock wave with properties predictable by the Rankine - Hugoniot equations. There are certain limitations due to the following conditions:

1. capacitor ringing in the driver section

2. impurity content from burnt mylar which coats the walls of the shock tube

3. jittering in the shock speeds from about 10 to 15 per cent, even when the bank voltage and downstream pressure are constant.

As a side note, we have often speculated whether the capacitor ringing effects may signify two different driving mechanisms; electromagnetic acceleration at low downstream pressures and electrothermal acceleration at high test gas pressures . 


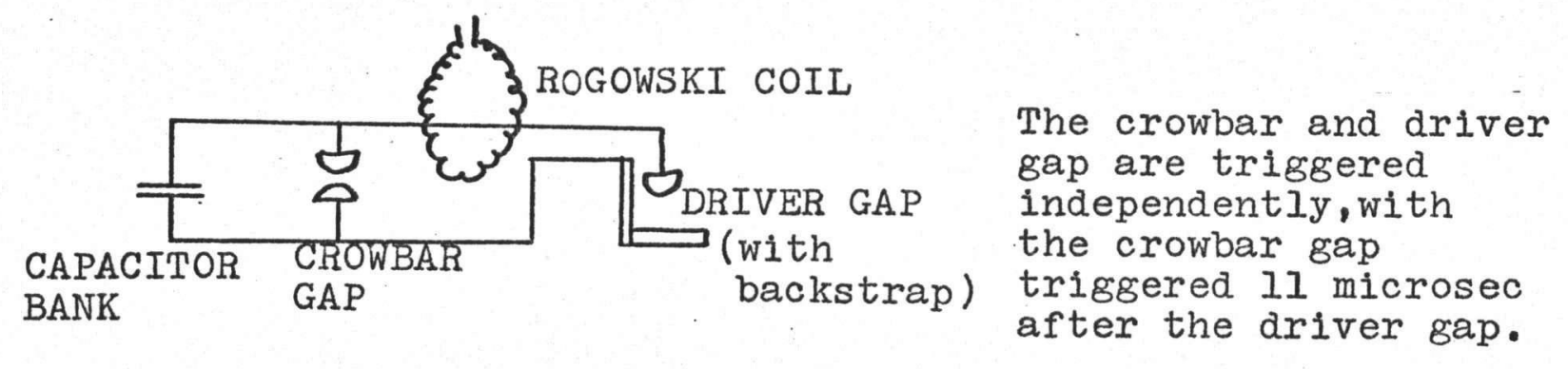

ROGOWSKI COIL MEASUREMENTS

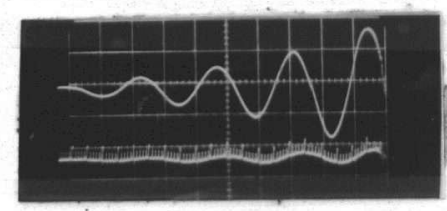

WITHOUT CROWBAR

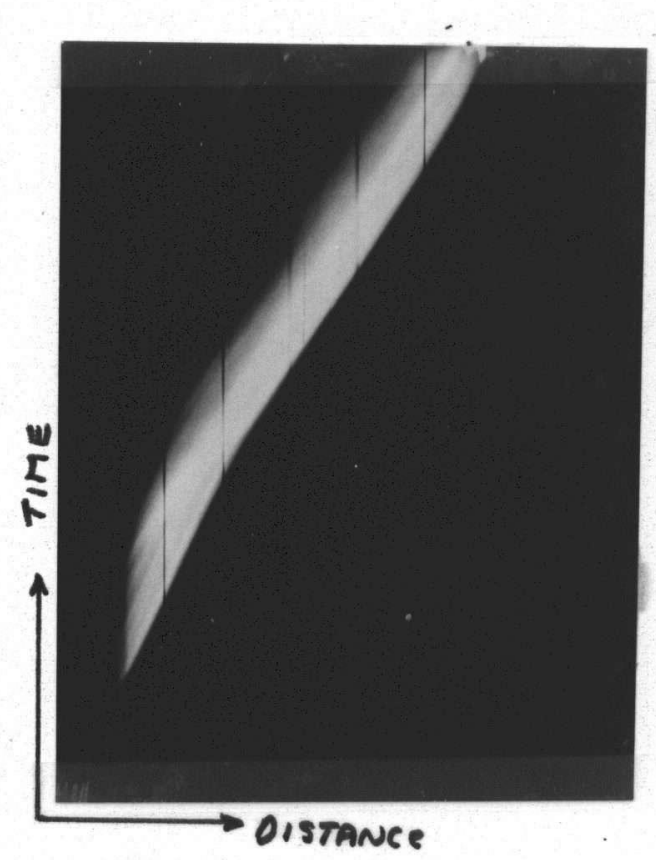

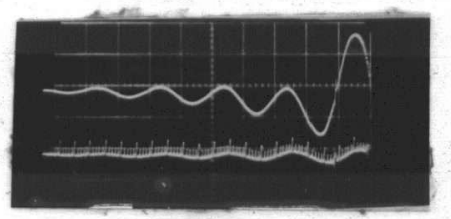

WITH CROWBAR

SMEAR PICTURE

(with crowbar)

CLEARLY INDICATING

THE CHANGE IN THE

DRIVING MECHANISM

Fig. D-8 Crowbar Results 


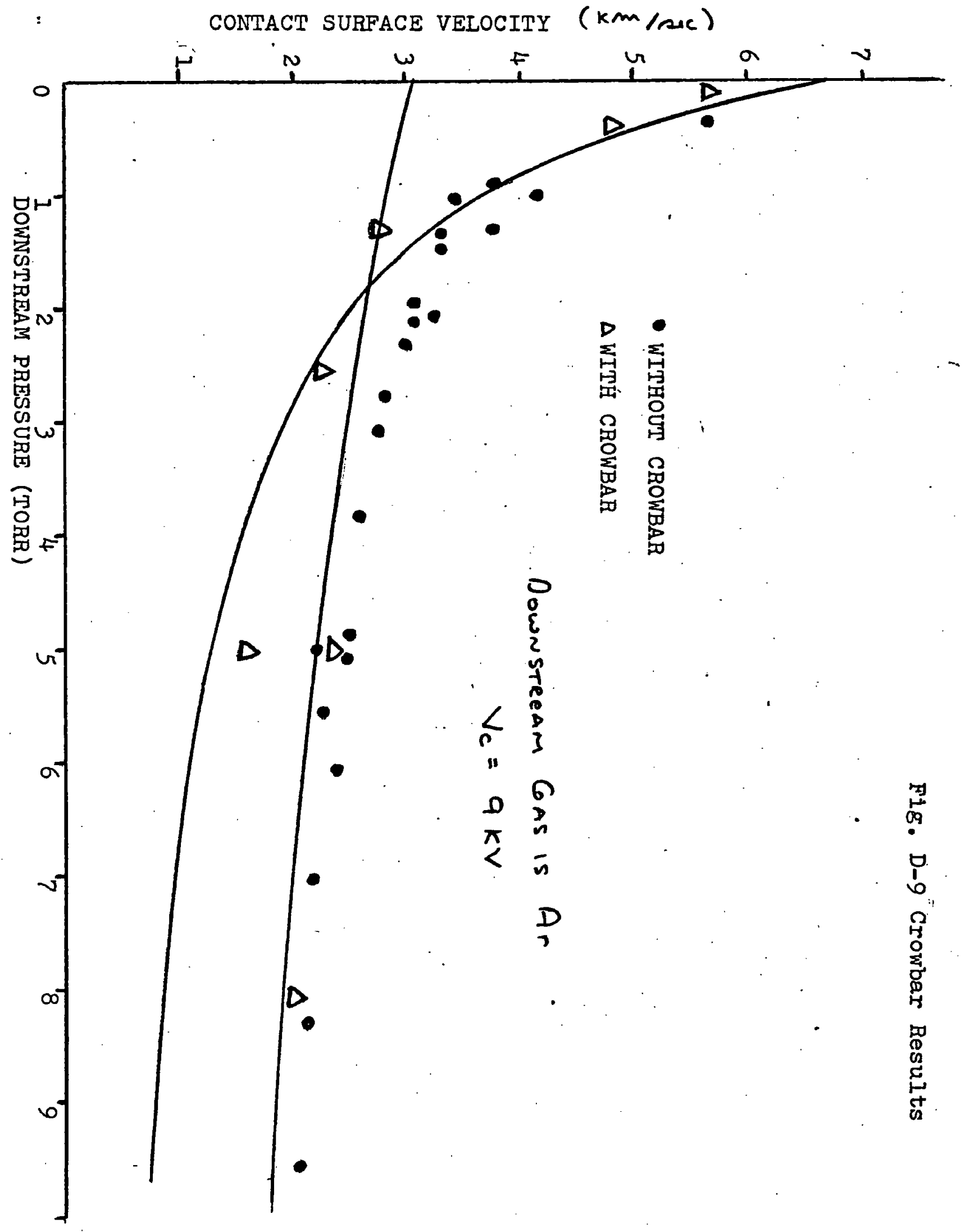


Appendix E

OTHER INTERESTING ASPECTS

Our experimental situation in itself is very interesting. We have noticed several phenomena which suggest another experiment.

One of our first observations was that under certain conditions $\left(\rho_{1}>5\right.$ TORR, $\left.M \sim 15\right)$, things would happen in the shock heated region of the known shock when the light source was turned on. Figure E-I is a smear picture which illustrates this phenomenon The luminous wedge starts when the light source is turned on. The effect seen in figure E-I is not reproducible in the sense that it is seen at every shot but it is predictable in the sense that whenever observed, it behaves in much the same way. There was always the same correlation between the start of the light source and the start of the luminous wedge.

Other similar Iuminous phenomena were also observed, although with even less reproducibility. When the shock front separated from the radiation front, a luminous wedge would appear in the known shock heated region (recall that the colour of the radiation front changed when the shock separated from 1t, fig. 9). Often times, instead of seeing a luminous wedge, a thin luminous line would appear some distance behind the known shock front. The luminous line appears very close to, and travels slightly slower than the contact surface. 


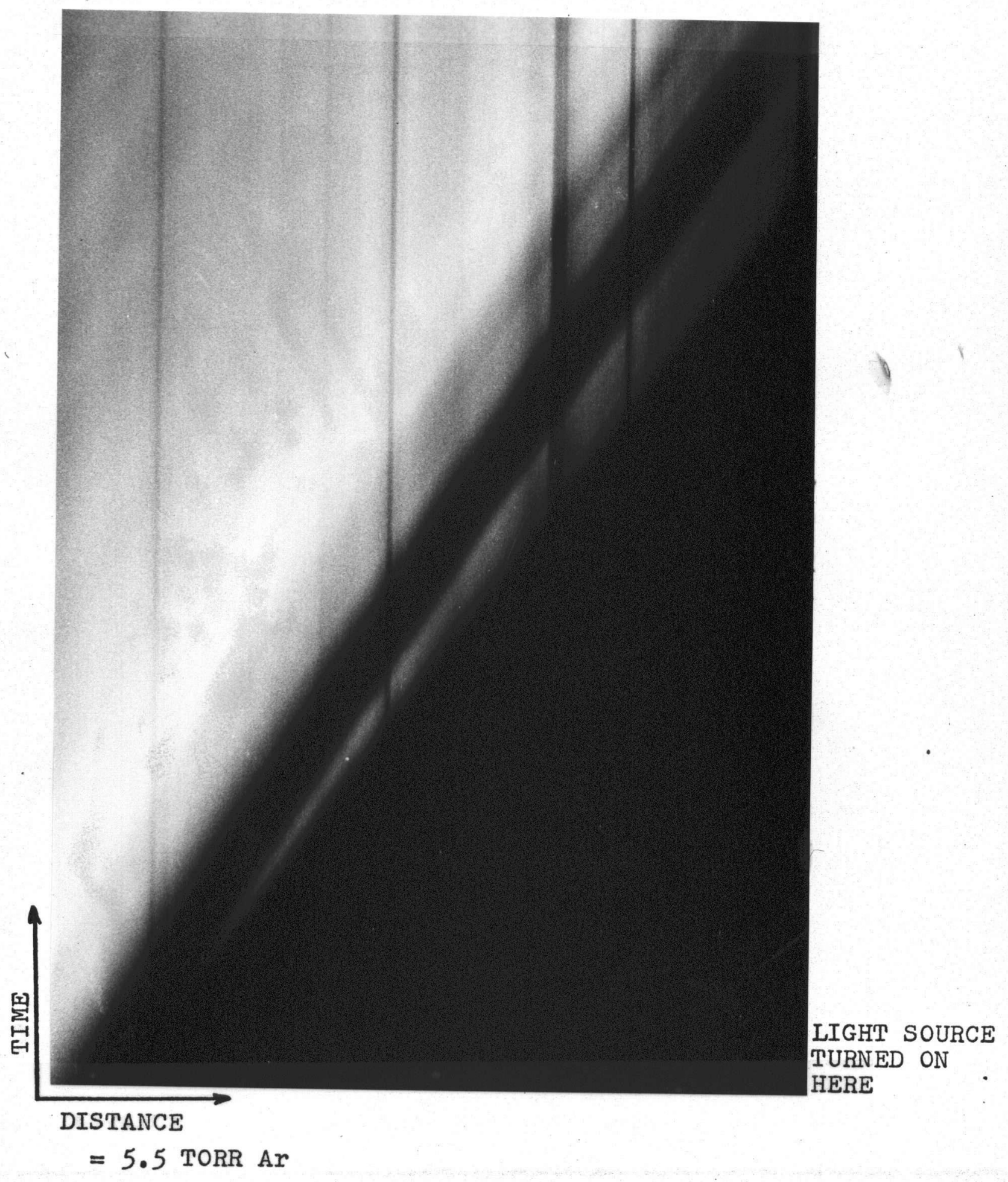

Fig. E-1 Radiation Effects in the Shock Heated Gas 
It appears obvious that these phenomena have something to do with the absorption of radiation in the shock heated gas. As a first guess, they could be radiation fronts, or excitation fronts, or luminoscity caused by some dynamical perturbation which is a result of the absorbed radiation.

This type of problem has been treated theoretically by R. G. Rehm (1968) and B. T. Chu (1955). Rehm followed the method of strained coordinates to calculate the flow field when laser radiation of small amplitude is absorbed over a finite region behind an established shock wave. He predicts the flow field as drawn in figure E-2. The main shock is expected to speed up, and a second shock is formed some time $T$ after the light is turned on and some distance behind the shock front.

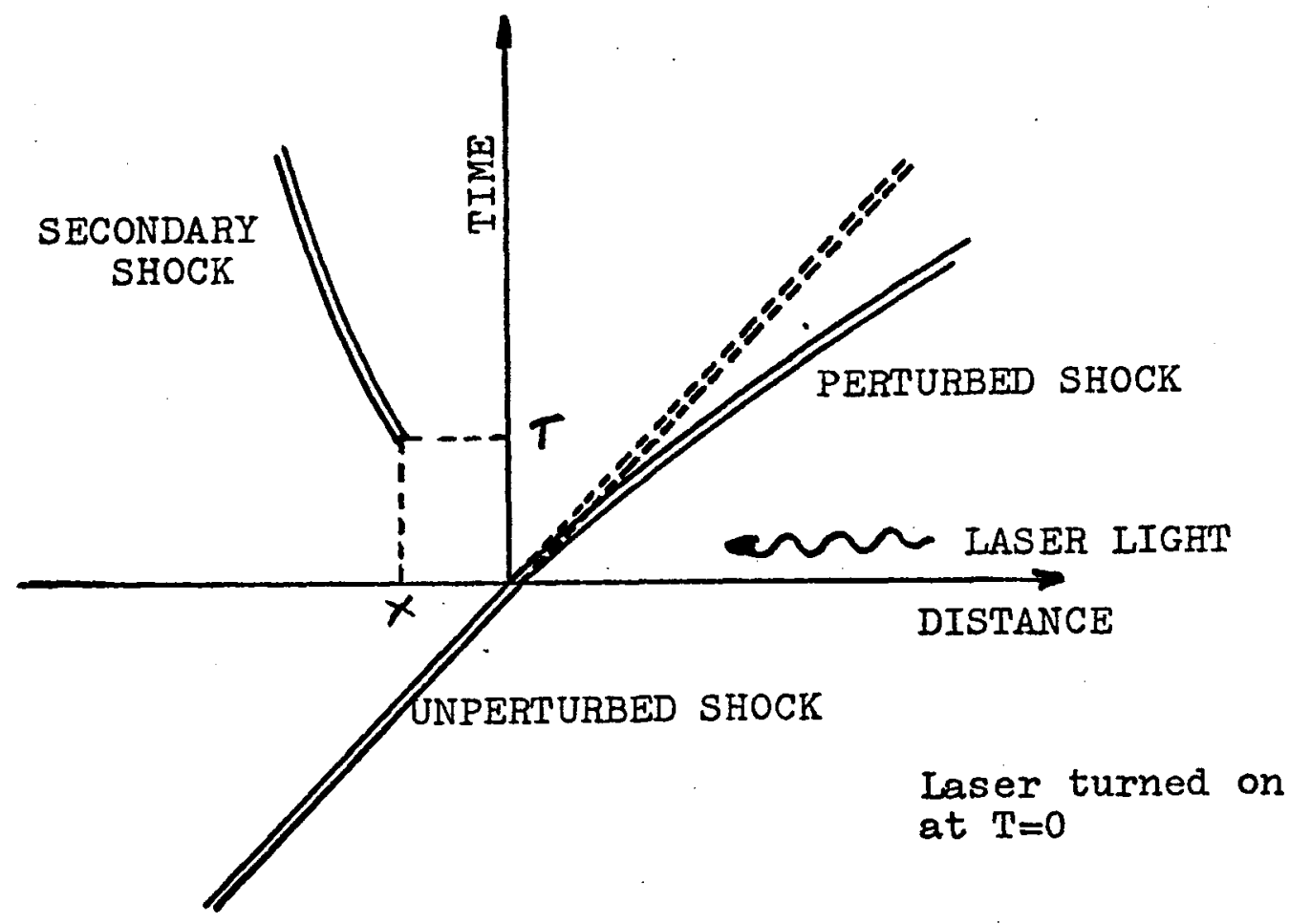

Fig. E-2 Radiative Energy Addition at a Shock Front 
Unfortunately, the accuracy of the velocity measurements from the smear pictures is not sufficient to measure the expected velocity increase. The expected secondary shock wave can be seen only on very few photographs, and then only by an observer who has faith in the theory. But something is happening and, to understand just what, could develop into an interesting experiment. 


\section{Appendix F}

THE EXPERIMENTAL SET-UP

The Bogan light source consists of an arc constricted through a narrow channel in a polyethylene rod. It radiates as a black body with an effective temperature of the order of $60,000^{\circ} \mathrm{K}$ for a period of about ten microseconds (Zuzak, 1968). The measuring device is a smear camera (J.P. Huni). The smear camera makes use of a rotating parabolic mirror driven by a high speed electric motor. When the motor is turned on, a pulse is generated every revolution and when the time between these pulses becomes as small as a prescribed time interval (sweep speed setting), a triggering pulse is sent out. The remaining triggering apparatus can have a jitter as high as tens of microseconds because of the large amount of time available on the film (of the order of 300 microseconds).

The triggering pulse is fed into a 162 Tektronix Waveform Generator which yields a negative sawtooth of several milliseconds duration. The negative sawtooth is fed into two 163 Tektronix Pulse Generators, each yielding pulses after variable and independent fractions of the sawtooth duration. These two pulse generators yield pulses which are delayed with respect to each other. Each pulse is fed into a separate thyratron and theophanis doubling agent which initiates the breakdown in the driver section of the shock tube and in an extermal series spark gap for the light source. The apparatus is 1llustrated in figures F-1 and F-2. 


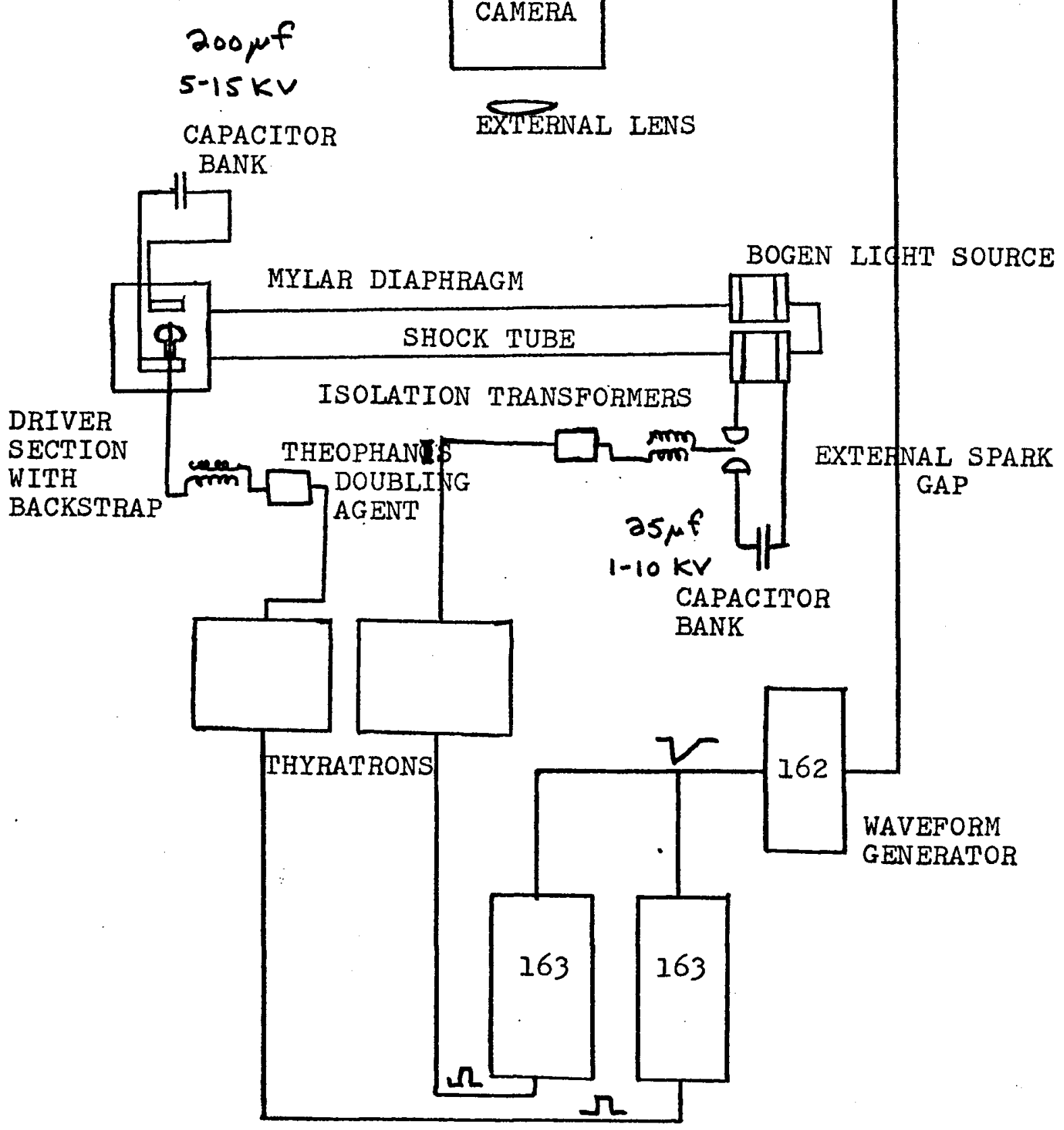

F1g. F-I Apparatus 


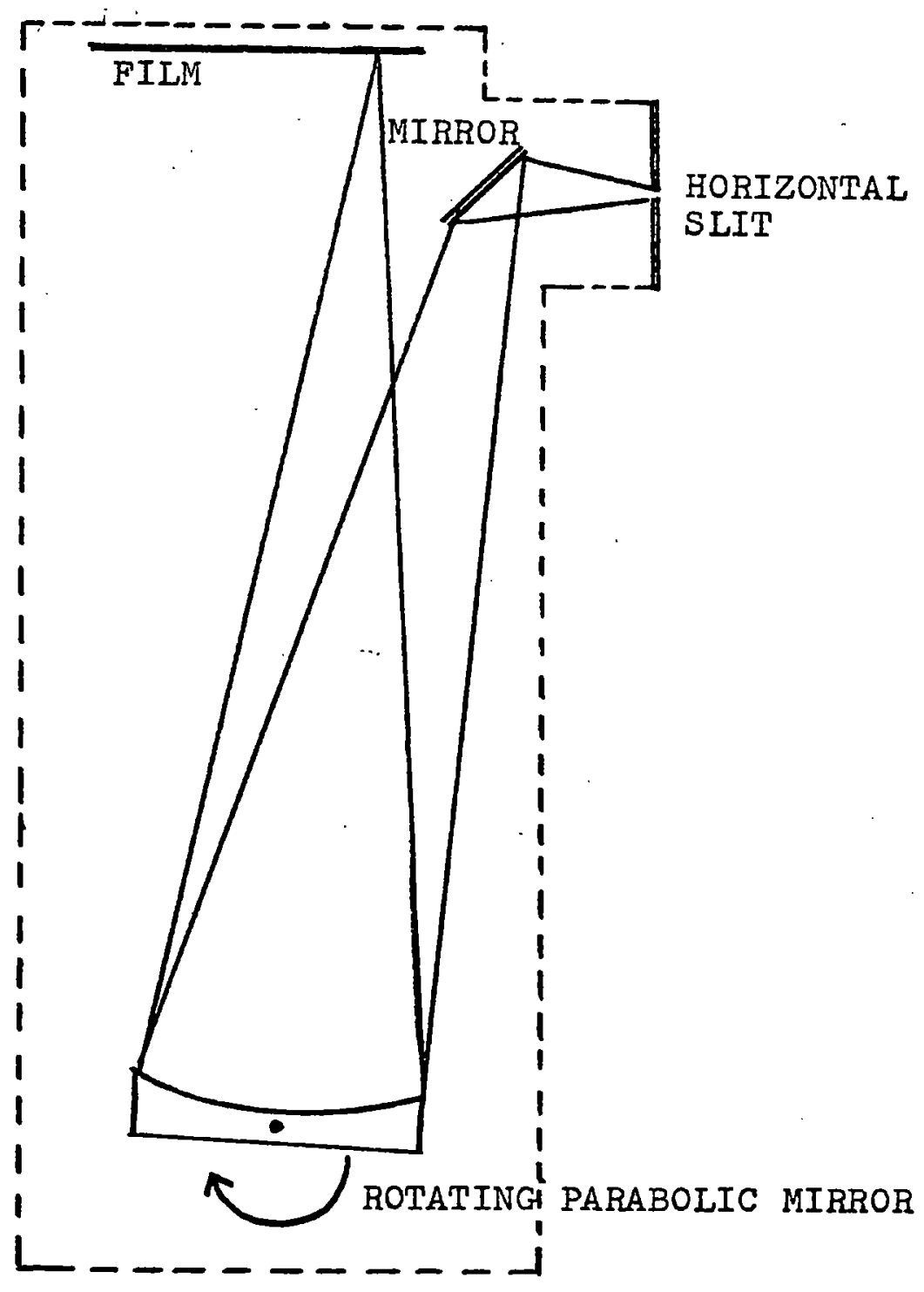

Fig. F-2 Smear Camera 
Appendix $G$

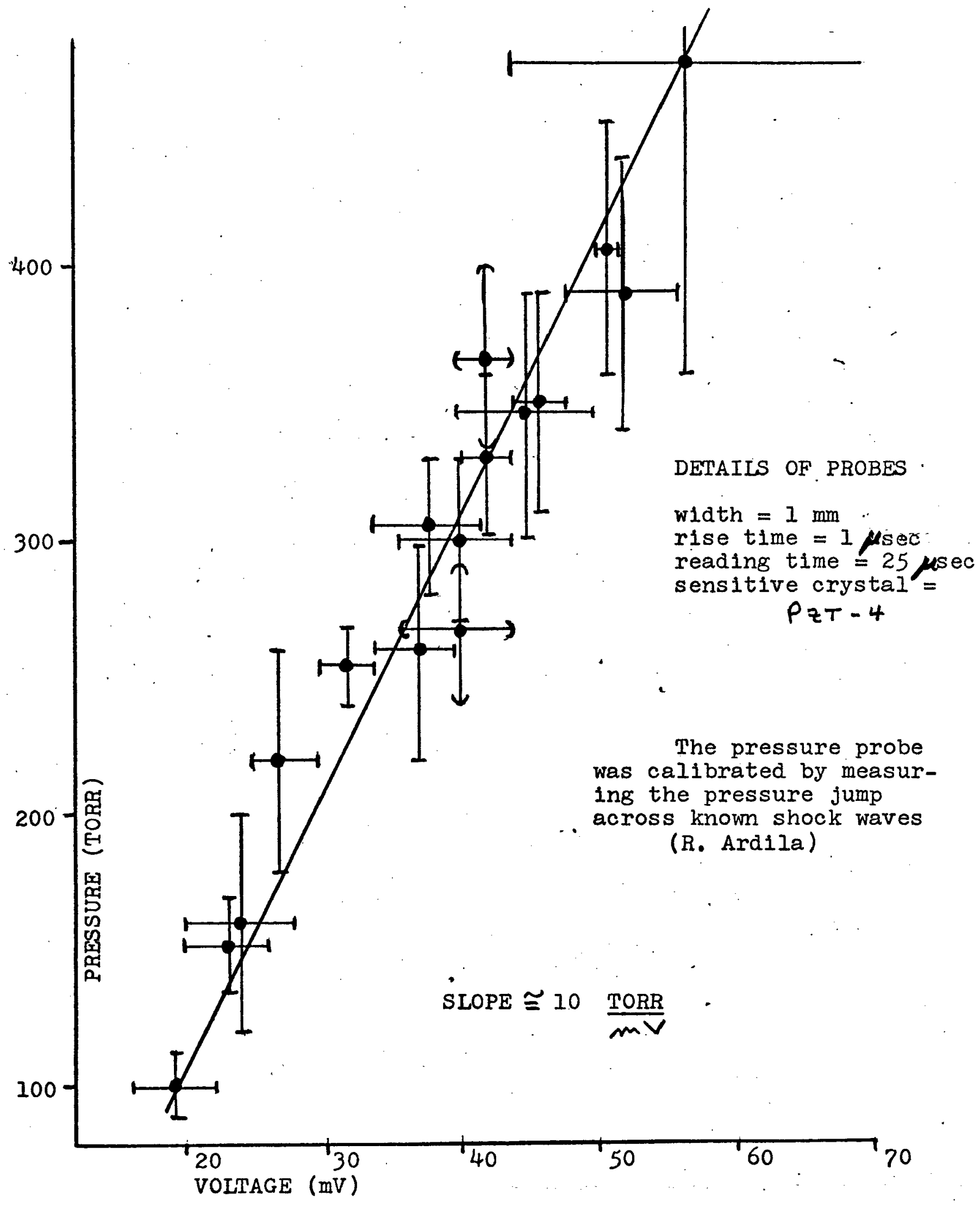

Fig. G Piezoelectric Probe Calibration 